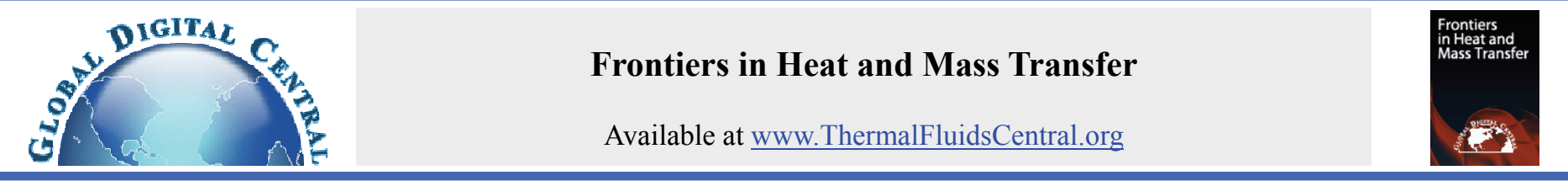

\title{
SYSTEMATIC STRATEGY FOR MODELING AND OPTIMIZATION OF THERMAL SYSTEMS WITH DESIGN UNCERTAINTIES
}

\author{
Po Ting Lin, Hae Chang Gea, Yogesh Jaluria* \\ Mechanical and Aerospace Engineering, Rutgers University, Piscataway, New Jersey, 08854, USA
}

\begin{abstract}
Thermal systems play significant roles in the engineering practice and our lives. To improve those thermal systems, it is necessary to model and optimize the design and the operating conditions. More importantly, the design uncertainties should be considered because the failures of the thermal systems may be very dangerous and produce large loss. This review paper focuses on a systematic strategy of modeling and optimizing of the thermal systems with the considerations of the design uncertainties. To demonstrate the proposed strategy, one of the complicated thermal systems, Chemical Vapor Deposition (CVD), is simulated, parametrically modeled, and optimized. The operating conditions, inlet velocities and susceptor temperatures, are the most significant factors in the CVD and are chosen as the design variables. Several responses - including the percentage of the working area, the mean of the deposition rate, the root mean square of the deposition, and the surface kurtosis - are chosen based on the physical needs and statistical foundations, and are utilized to represent the productivity and the quality of the thin-film deposition. One of the Response Surface Method (RSM), the Radial Basis Function (RBF), is employed to formulate the objective and constraint functions for the optimization. However, it is not until the design uncertainties are considered that the thermal system designs have high risk of the violations of the performance constraints. The Reliability-Based Design Optimization (RBDO) algorithms are used to solve the optimization problems with the design uncertainties. The most famous RBDO methods are the Reliability Index Approach (RIA) and the Performance Measure Approach (PMA). In RBDO, probabilistic constraints are established with respect to either normally or non-normally distributed random variables. The optimal solutions are found subjected to the allowable level of the failure probabilities. The Monte Carlo Simulation (MCS) results can be used to evaluate the failure probabilities. As a result, the proposed systematic strategy of parametrically modeling and optimizing with design uncertainties can be applied to either experiments or simulations of other thermal systems to quantitatively represent the performances, improve their productivity, maintain the quality control, and reduce the probability of the system failure.
\end{abstract}

Keywords: Chemical Vapor Deposition (CVD), Response Surface Method (RSM), Radial Basis Function (RBF), Reliability-Based Design Optimization (RBDO), Reliability Index Approach (RIA), Performance Measure Approach (PMA)

\section{INTRODUCTION}

Thermal systems not only are essential technologies in engineering practice but also play significant roles in our lives. With the continuously growing needs of the thermal systems in many different applications, such as power systems, air conditioning, energy conversion, chemical processing, material processing, aerospace, and automobiles, the design and the optimization of the thermal systems have become very important research works in the engineering field.

The thermal systems are often very complicated because of the complex physics and mechanics involved in the systems, including fluidic mechanics, heat transfer, mass transfer, and chemical reactions. It is nearly impossible to realize the closed-form relationships between the system performances and all the involved variables. Therefore, it is important to firstly understand the basic characteristics of the thermal systems and subsequently determine the principal design variables which dominantly control the system performances.

A systematic strategy is then desired to model and optimize the thermal systems. This proposed strategy must be able to resolve the following questions:
- How to model the system performances in terms of the design variables so that the system performances can be accurately described by the proposed models?

- How to formulate the optimization problems in terms of the defined models for improving the system performances?

In the aspect of the modeling, the mathematical models that are able to quantitatively and literally represent the physical behaviors of the system performances are necessary. With the desired models, the optimization problems can then be formulated to achieve the goals of the thermal system designs.

Unfortunately, the existence of the design uncertainties is unavoidable. A traditional deterministic optimization algorithm often leads the optimal solution to the boundaries of the active constraints. Without the considerations of the design uncertainties, the optimal solution from the deterministic optimization formulation is unreliable and has high probabilities of violating the active constraints. Therefore, additional attentions should be drawn to the optimization problems with the design uncertainties and the following questions should be answered:

- How to formulate a non-deterministic optimization problem when the design variables are uncertain?

\footnotetext{
* Corresponding author. Email: jaluria@jove.rutgers.edu
} 
- How to solve this non-deterministic optimization problem and how to solve it efficiently?

\section{THERMAL SYSTEMS}

As described earlier, the thermal systems are very important in various applications and our lives. To design and optimize the thermal systems, the most fundamental step is to recognize their existence and classify them into different groups in terms of their functionalities. A common classification of the thermal system is given as follows (Jaluria, 1998):

- Manufacturing and materials processing systems.

- Energy systems.

- Cooling systems for electronic equipment.

- Environmental and safety systems.

- Aerospace systems.

- Transportation systems.

- Air conditioning, refrigeration, and heating systems.

- Fluid flow systems and equipment.

- Heat transfer equipment.

- Other thermal systems.

After understanding the physics and the mechanics behind the thermal systems, the control variables are chosen to determine the system performances. Therefore, the decision of the design variables is the key factor of the modeling and the optimization of the thermal systems.

Manufacturing and Materials Processing Systems: Heat transfer plays an important role in the manufacturing and materials processing systems, where the materials often change their mechanical properties due to temperature changes. The controls of the temperature changes determine the productivity and the quality of the processes. Examples include crystal growing, metal casting, metal forming, plastic injection molding, etc. (Jaluria, 1998) One of those processes, the Chemical Vapor Disposition (CVD) process, will be considered in the later sessions. A systematic strategy to design and optimize the CVD process with design uncertainties will be proposed and it can be applied to the design and the optimization of other thermal systems.

Energy Systems have become one of the most important thermal systems in recent years in which the thermodynamics of the energy conversions are the issues of most concern. Energy systems are often very complicated because they contain several subsystems, such as energy collector, steam generator, turbines, condenser, etc. (Van Wylen and Sonntag, 1986) Numerous design variables should be considered to improve the thermal efficiency of the energy system.

Cooling Systems are essentially important for electronic equipment where the operating temperatures are constrained within certain allowable temperatures (Steinberg, 1980). Other constraints for the cooling systems include the spatial working space, the allowable noise, etc. The objective of the cooling system design is often minimizing the ratio of used power to reduced temperature, while the design variables are often the geometries of the heat sink which differ the surface area of the heat transfer (Yang and Peng, 2008; 2009).

Environmental and Safety Systems: Safety is an important factor for systems with extreme environmental conditions, such as high temperature and toxicity. Environmental and safety systems include the applications for heat rejection to air or water, control of the temperature and the pollution of thermal systems, etc. The heat rejection from a power plant is a good example while the heat is dumped to the river as a cooling pond. The operation of the power plant will be under high risks if the safety systems fail. Therefore, the safety system should be taken into careful consideration in the design of the systems with extreme environmental conditions.
Aerospace Systems: Thermal systems are the most important components in aerospace systems, such as rockets, turbines, etc. For an instance of the rocket system, the alcohol/water mixture is pumped into the combustion chamber to heat the fuel and cool the chamber. The balance between the high thrust for launching and the efficient cooling is the focus in the design of the rocket systems.

Transportation Systems cannot operate without the existence of the thermal systems, including diffusion, compression, combustion, turbine, and nozzle systems. In a turbine engine, the thrust energy comes from the combustion of the air/fuel mixture. Thermodynamics is significant for the design of the transportation systems (Van Wylen and Sonntag, 1986), while they are often optimized in terms of maximizing the ratio of the generated power to utilized fuel.

Air conditioning, Refrigeration, and Heating Systems are indispensable to our daily lives. Detailed information about such kind of the thermal systems can be found in the references of (Stoecker and Jones, 1982; Kreider and Rabl, 1994). Take the air conditioning system as an example, the physical phase, the temperature, and the pressure of the fluid change via the mechanisms of the condenser, the evaporator, and the compressor. The optimization for such thermal systems often focuses on decreasing the power consumption and improving the efficiency of the temperature control.

Fluid Flow Systems and Equipment include hydraulic components, such as pumps, turbines, compressors, fans, etc. Fluid mechanics is of the major concern in the fluid flow systems and is closely related to the thermal systems with energy transmission, cooling, and mass transfer (Fox and McDonald, 1992). One of the examples is a wastewater treatment system in which the waste water is transferred through several different fluid mechanisms, clarified, and delivered to the drainage system.

Heat Transfer Systems contain heat exchangers (Kays and London, 1984), furnaces, heaters, condensers, etc. The most straightforward example is the heat exchangers where the heat is transferred to the water to increase its temperature for human usage. The design of the heat exchanging mechanisms considers the transmission of the energy and the control of the heat loss.

There is never a best way to classify all kinds of the thermal system but the most practical ones have been covered and discussed. In the later session, one of the materials processing systems, the CVD process, is taken into consideration. The design and the optimization of the CVD process will be studied and a systematic strategy will be proposed to implement the modeling and the optimization of the CVD process with design uncertainties. The proposed methodology is expected to have the capability of designing and optimizing of the other thermal systems.

\section{Chemical Vapor Deposition Processes}

Chemical Vapor Deposition (CVD) is a process that a solid crystalline or amorphous layer is formed on a heated substrate by chemically reacting premixed gases using the activation energy. The Fig. 1 shows a schematic sequence of the steps of the CVD process. The process involves the transport of reactants to the susceptor, the chemical reactions and generations of new species, the desorption of the reaction products, and their diffusion back to the main stream (Mahajan, 1996). Unlike the cold substrate in the Physical Vapor Deposition (PVD) process (Fraser, 1983; Foxon, 1994), the one in the CVD process has a higher temperature with a various range from 500 to $1500 \mathrm{~K}$ due to different applications and materials, different types of CVD processes, and different configurations of the CVD reactors. The details about the applications of the CVD processes, different types of the CVD processes, and the configurations of the CVD reactors will be discussed in the following subsession. 


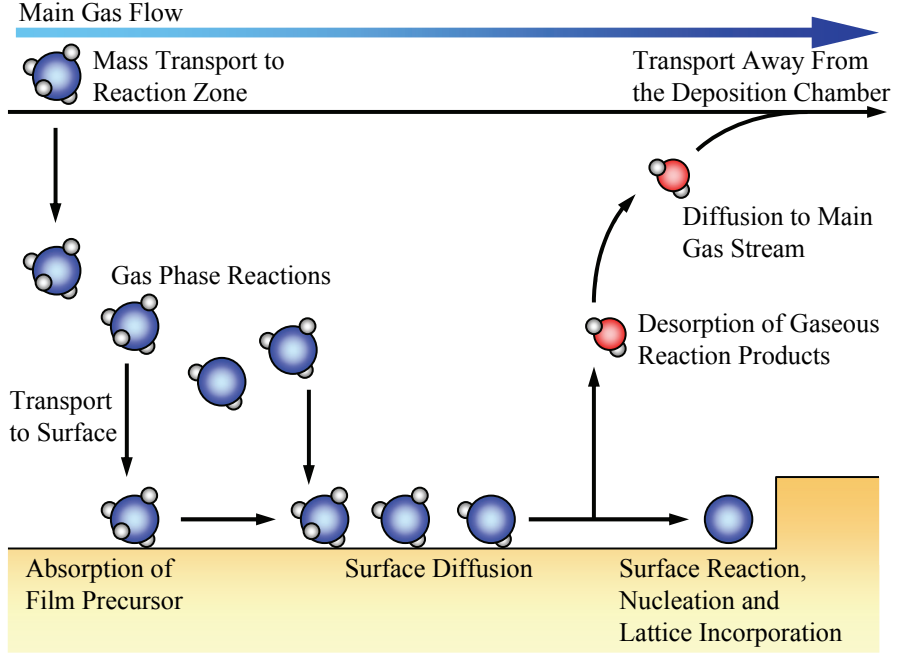

Fig. 1 Schematic sequence of steps in CVD process (Mahajan, 1996).

\subsection{Different Applications of the CVD Processes}

The CVD-based products have become more and more important in our daily lives because of the high quality of the deposited thin layers with various kinds of materials. The CVD process is well utilized to produce highly uniform thin films deposited on different kinds of substrates (typically 0.01 to $10 \mu \mathrm{m}$ ) (Mahajan, 1996). It is used in a wide range of applications where thin coatings of high purity are required.

The CVD processes can be utilized to produce high-quality microstructures in semiconductors, special materials with dielectric properties as insulators, and metallic conductors with different resistivities. Furthermore, the CVD processes generate high-strength products such as protective coatings, anticorrosive coatings, and ceramic materials. Besides the productions of thin layers, it is possible to generate powers and fibers of different materials by the CVD processes. Other applications such as optical materials and synthetic diamonds have high qualities and purities of different materials. The common materials in different applications of the CVD processes are listed in Table 1. Most of the research interests in the later discussions is directed at the CVD of silicon because of its relevance to the semiconductor industry (Gardeniers et al., 1989; Breiland and Coltrin, 1990).

\subsection{Different Types of the CVD Processes}

CVD processes can be classified in terms of their operating conditions or different kinds of instruments. With different operating pressures of the CVD reactors, the CVD processes include three different types:

- Atmospheric Pressure CVD (APCVD).

- $\quad$ Low-Pressure CVD (LPCVD).

- Ultra-High Vacuum CVD (UHVCVD)

The APCVD operates at the pressure of 0.1 to $1 \mathrm{~atm}$ while the LPCVD works at a lower pressure of $10^{-3} \mathrm{~atm}$ (Mahajan, 1996). Other modern CVD processes reach high or ultra-high vacuum (below $10^{-6} \mathrm{~Pa}$ ) and have high-quality thin-film depositions.
Table 1 Common materials in different applications of the CVD processes (Sherman, 1987; Gardeniers et al., 1989; Breiland and Coltrin, 1990; Fotiadis et al., 1990; Creighton and Parmeter, 1993; Gladfelter, 1993; Rebenne and Bhat, 1994; Cheng et al., 1995; Hintermann, 1996; Mahajan, 1996).

\begin{tabular}{|c|c|}
\hline $\begin{array}{l}\text { Different CVD } \\
\text { Applications }\end{array}$ & Common Materials \\
\hline Semiconductors & silicon $(\mathrm{Si})$, gallium arsenide $(\mathrm{GaAs})$ \\
\hline Dielectrics & silicon dioxide $\left(\mathrm{SiO}_{2}\right)$, silicon nitride $\left(\mathrm{Si}_{3} \mathrm{~N}_{4}\right)$ \\
\hline Metallic conductors & $\begin{array}{l}\text { tungsten silicide }\left(\mathrm{WSi}_{2}\right) \text {, molybdenum } \\
\text { silicide }\left(\mathrm{MoSi}_{2}\right) \text {, tungsten }(\mathrm{W}) \text {, aluminum } \\
(\mathrm{Al}) \text {, molybdenum }(\mathrm{Mo}), \text { polysilicon }(\mathrm{Si})\end{array}$ \\
\hline Protective coatings & $\begin{array}{c}\text { titanium nitride }(\mathrm{TiN}), \text { Tungsten }(\mathrm{W}) \\
\text { molybdenum }(\mathrm{Mo}) \text {, gold }(\mathrm{Au}) \text {, platinum }(\mathrm{Pt})\end{array}$ \\
\hline Ceramics & $\begin{array}{c}\text { aluminum oxide }\left(\mathrm{Al}_{2} \mathrm{O}_{3}\right) \text {, titanium carbide } \\
(\mathrm{TiC}) \text {, silicon carbide }(\mathrm{SiC}) \text {, boron carbide } \\
\left(\mathrm{B}_{4} \mathrm{C}\right) \text {, titanium biboride }\left(\mathrm{TiB}_{2}\right)\end{array}$ \\
\hline $\begin{array}{l}\text { Anticorrosive } \\
\text { coatings for turbine } \\
\text { blades } \\
\end{array}$ & $\begin{array}{l}\text { boron nitride }(\mathrm{BN}) \text {, molybdenum disilicide } \\
\left(\mathrm{MoSi}_{2}\right) \text {, silicon carbide }(\mathrm{SiC}) \text {, boron carbide } \\
\left(\mathrm{B}_{4} \mathrm{C}\right)\end{array}$ \\
\hline $\begin{array}{l}\text { Powers for sintering } \\
\text { and hot pressing }\end{array}$ & silicon nitride $\left(\mathrm{Si}_{3} \mathrm{~N}_{4}\right)$, silicon carbide $(\mathrm{SiC})$ \\
\hline $\begin{array}{c}\text { Fibers for } \\
\text { composite materials }\end{array}$ & $\begin{array}{c}\text { boron }(\mathrm{B}), \text { boron carbide }\left(\mathrm{B}_{4} \mathrm{C}\right) \text {, silicon } \\
\text { carbide }(\mathrm{SiC})\end{array}$ \\
\hline $\begin{array}{l}\text { High-purity } \\
\text { monolithic } \\
\text { materials for } \\
\text { infrared optics } \\
\end{array}$ & $\begin{array}{c}\text { zinc selenide }(\mathrm{ZnSe}), \text { zinc sulfide }(\mathrm{ZnS}) \text {, } \\
\text { cadmium sulfide }(\mathrm{CdS}) \text {, cadmium telluride } \\
(\mathrm{CdTe})\end{array}$ \\
\hline Synthetic diamonds & carbon $(\mathrm{C})$ \\
\hline
\end{tabular}

Table 2 Different types of the CVD processes.

\begin{tabular}{|c|c|}
\hline $\begin{array}{c}\text { Different } \\
\text { Types }\end{array}$ & Descriptions \\
\hline $\begin{array}{l}\text { Plasma- } \\
\text { Enhanced } \\
\text { CVD } \\
\text { (PECVD) }\end{array}$ & $\begin{array}{l}\text { A CVD instrument with plasma enhancement where } \\
\text { higher density of reactant species are produced to the } \\
\text { substrate due to the high-energy electron impact. } \\
\text { Higher activity of the gaseous species allows } \\
\text { deposition at comparatively low temperature ( } 450 \text { to } \\
650 \mathrm{~K}) \text {. (Sherman, 1987; Mahajan, 1996) }\end{array}$ \\
\hline $\begin{array}{l}\text { Metal- } \\
\text { Organic } \\
\text { CVD } \\
\text { (MOCVD) }\end{array}$ & $\begin{array}{l}\text { Also known as Organo-Metallic Vapor Phase Epitaxy } \\
\text { (OMVPE). An epitaxial growth of materials from } \\
\text { the surface chemical reaction of organic or metal- } \\
\text { organic compounds and an important process for the } \\
\text { manufacturing of solar cells and LEDs. (Stringfellow, } \\
\text { 2001; Kurtz et al., 2007) }\end{array}$ \\
\hline $\begin{array}{c}\text { Laser CVD } \\
\text { (LCVD) }\end{array}$ & $\begin{array}{l}\text { A laser-assisted instrument that locally heat the } \\
\text { substrate to activate the CVD reaction with precise } \\
\text { control. (Allen, 1981) }\end{array}$ \\
\hline $\begin{array}{c}\text { Photo CVD } \\
\text { (PCVD) }\end{array}$ & $\begin{array}{l}\text { A photo-assisted deposition technique where UV or } \\
\text { visible photon energies are used for gas } \\
\text { decomposition. The deposition at very low } \\
\text { temperature ( } 300 \text { to } 450 \mathrm{~K} \text { ) is allowed but having a } \\
\text { low deposition rate and poor uniformity. (Eden, 1991; } \\
\text { Mahajan, 1996) }\end{array}$ \\
\hline $\begin{array}{l}\text { Chemical } \\
\text { Vapor } \\
\text { Infiltration } \\
(\mathrm{CVI})\end{array}$ & $\begin{array}{l}\text { A variant CVD device that deposits within a porous } \\
\text { body and is widely used for the fabrication of } \\
\text { ceramic materials. (Naslain and Langlais, 1986) }\end{array}$ \\
\hline $\begin{array}{l}\text { Hot Wire } \\
\text { CVD } \\
\text { (HWCVD) }\end{array}$ & $\begin{array}{l}\text { A special instrument for producing high-temperature } \\
\text { gas decomposition but room-temperature deposition } \\
\text { on the substrate. (Lau et al., 2001) }\end{array}$ \\
\hline $\begin{array}{l}\text { Atomic } \\
\text { Layer CVD } \\
\text { (ALCVD) }\end{array}$ & $\begin{array}{l}\text { A technology to produce ultrathin layers of } \\
\text { crystalline materials (typically } 1 \text { to } 50 \mathrm{~nm} \text { ). (Nilsen, } \\
\text { 2003) }\end{array}$ \\
\hline
\end{tabular}


Another method to classify the CVD processes considers the operating wall temperature of the CVD chamber. They are:

- Cold-Wall CVD.

- Hot-Wall CVD.

Most of the CVD processes operate with the hot-wall reactors and the gaseous temperature is distributed uniformly inside the reactor. The advantage of the hot-wall setting is higher deposition rate and better uniformity of the deposition. On the other hand, the cold-wall setting allows higher throughput and easier cleaning but has lower speed of the deposition and poor uniformity of the thin film.

In the consideration of different instruments, a wide variety of CVD processes have been developed, listed in Table 2. Plasmaassisted CVD processes operate at low pressure and allow the cold-wall setting because the plasma bombards the gas mixture and decompose into active species for deposition at low temperature. Photo CVD is another instrument that works at low temperature and uses the activation energy from ultraviolet or visible photons to achieve the gaseous decomposition. Laser CVD is a device that provides higher activation energy and, furthermore, very accurate control of the local deposition. Other CVD instruments like Metal-Organic CVD (MOCVD) and Chemical Vapor Infiltration (CVI) have specific applications. The epitaxial growths of III/V materials from MOCVD have become very important in the manufacturing of solar cells and light-emitting diodes (LEDs), and the semiconductors with organometallic compounds. CVI is the specific instrument for the growth of ceramic materials in a porous body.

\subsection{Different Configurations of the CVD Reactors}

For different operating conditions and applications, several different configurations of the CVD reactors have been developed. The Fig. 2 illustrates some common CVD reactors. Typically, the reactors in Fig. 2 (a), (b), and (c) are utilized for cold-wall settings and the ones in Fig. 2 (d), (e), and (f) have higher wall temperatures of the CVD chambers. The barrel reactor has been greatly used for the hugeamount production of the silicon epitaxial wafers. The vertical reactor with rotating susceptor is often utilized for single-wafer depositions; on the other hand, the one in Fig. 2 (e) has higher area of uniform deposition and is used for the depositions of multiple wafers. The tubular reactor is usually used to deposit films with polysilicon and dielectric materials. In the later discussion, the modeling and the optimization of the CVD processes focus on the configuration of the vertical impinging reactor with the stationary susceptor in Fig. 2 (e).

In the design and optimization of the CVD processes, different design variables should be taken into consideration for different configurations. For example, the horizontal reactor in Fig. 2 (a) has a tilt angle of the susceptor for uniform deposition with horizontal gaseous flow of the reactant species. The rotation speeds of the reactors in Fig. 2 (c) and (d) differ the quality of the deposition. The directions of the reactant flow above the susceptor certainly provide different characteristics of the fluidic mechanics and heat transfer. Among all configurations of the CVD reactors, there are some common variables that dominate the performance of the thin-film deposition, including the concentration of the gaseous reactant in the inlet flow, the velocity of the inlet flow, the temperature of the susceptor, the temperature of the chamber wall, the operating pressure in the CVD chamber, etc. The review about the design of the CVD processes will be given in the next subsession.

\section{Design of the CVD Process}

Different designs of the CVD processes have a wide variety of the film thickness, generally ranges from a few nanometers to tens of microns. As described previously, the film formation process is highly dependent on the flow and the heat transfer between the gas and the heated substrate. Therefore, in order to produce thin films with higher deposition rates and quality, the operation conditions must be studied.
There are two major aspects to be considered in the design of the CVD processes:

- $\quad$ Experiments or simulations of the CVD processes.

- Modeling of the responses.

Reviews about those aspects are given in the following.

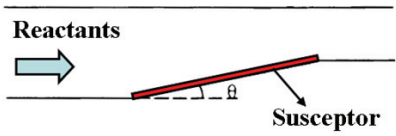

(a)

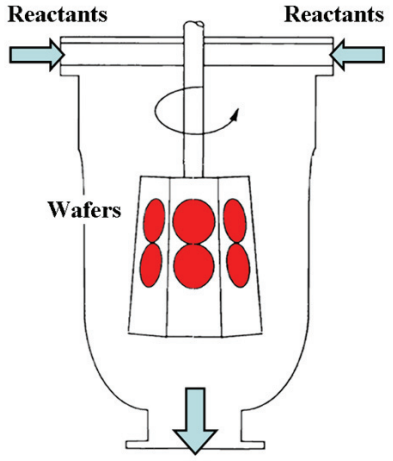

(c)

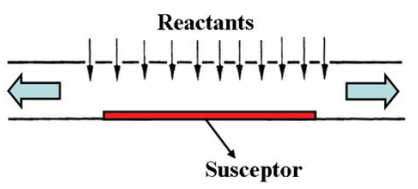

(e)

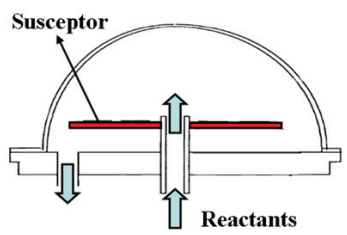

(b)

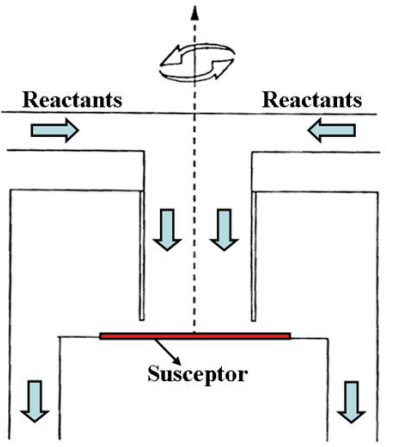

(d)

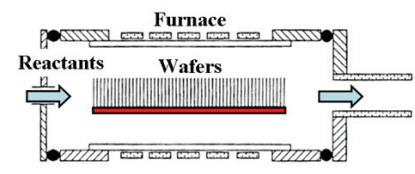

(f)
Fig. 2 Different configurations of the CVD reactors: (a) horizontal reactor, (b) pancake reactor, (c) barrel reactor, (d) vertical impinging reactor with rotating susceptor, (e) vertical impinging reactor, and (f) tubular reactor. (Mahajan, 1996)

Table 3 Typical design variables and responses in the CVD designs. (Dimitrios et al., 1987; Fotiadis et al., 1990; Jensen et al., 1991; Mahajan, 1996; Chiu and Jaluria, 2000; Chiu, Richards et al., 2000)

\begin{tabular}{|c|c|c|}
\hline \multicolumn{2}{|r|}{ Design Variables } & Responses \\
\hline $\begin{array}{c}\text { Hardware } \\
\text { settings }\end{array}$ & $\begin{array}{l}\text { - Susceptor size } \\
\text { - Shape of CVD chamber } \\
\text { - Angle of susceptor vs. } \\
\text { the flow direction } \\
\text { - Orientation of } \\
\text { susceptor vs. gravity } \\
\text { - Buoyancy driven / } \\
\text { force driven flow } \\
\text { - Diffusivity of susceptor } \\
\text { material }\end{array}$ & $\begin{array}{l}\text { - Deposition thickness } \\
\text { - Deposition rate } \\
\text { - Deposition uniformity } \\
\text { - Nusselt number } \\
\text { - Temperature } \\
\text { distribution of the } \\
\text { susceptor } \\
\text { - Purity of the deposition } \\
\text { - Composition of the } \\
\text { deposition }\end{array}$ \\
\hline $\begin{array}{l}\text { Operating } \\
\text { conditions }\end{array}$ & $\begin{array}{l}\text { - Velocity of inlet flow } \\
\text { - Susceptor temperature } \\
\text { - Operating pressure } \\
\text { - Rotation speed of the } \\
\text { susceptor } \\
\text { - Concentration of the } \\
\text { reactant species }\end{array}$ & $\begin{array}{l}\text { - Adhesion to the } \\
\text { substrate } \\
\text { - Surface morphology } \\
\text { - Grain structure in the } \\
\text { deposition } \\
\text { - Distance of flow } \\
\text { separation }\end{array}$ \\
\hline
\end{tabular}




\subsection{Design Variables and Responses of the CVD Processes}

Once the reactant species, the type of the CVD process, and the CVD configuration have been determined for the desired thin-film production on the susceptor, several operating parameters should be chosen to perform the experiments or the simulations of the CVD process. Among those parameters, some of them dominate the control of the deposition performance and are selected to be the design variables. The typical design variables are categorized into two different types, including hardware settings and operating conditions, and listed in Table 3. The hardware settings vary the boundary conditions and the mechanical properties of the fluid mechanics and the heat transfer in the CVD processes. On the other hand, the operating conditions are the quantitative variables to control the behavior of the reactant fluid and the performance of the deposition. Besides the hardware and operating design variables, the rest of the parameters remain constant because of either their minor impacts to the deposition or the simplicity of the CVD design. In this research work, the inlet velocity and the susceptor temperature are chosen as the design variables because their quantities can be easily controlled by the designers.

The merit of the deposition performance requires several quantitative responses to judge, where those responses typically have physical meanings and provide numerical measures. Table 3 points out several common responses from either the experiments or the simulations of the CVD processes. Among those typical responses, some of them still lack of numerical measures to decisively quantify its degree of intensity. For example, the deposition uniformity itself is a subjective scale of the quality of the CVD production. George (George, 2007) utilized a weighted sum of the local slopes of the deposition to quantify its uniformity. Lin et al. (Lin, Jaluria et al., 2009) used some standard statistical measures, including the root mean square and the kurtosis, as the responses of the uniformity factors. More details about the chosen design variables and the significant responses will be discussed in the later session.

\subsection{Experiments or Simulations of the CVD Processes}

The mechanics of the CVD process, in which the flow, the heat transfer, and the chemical reaction are involved, is very complicated. The flow in the CVD process has firstly been visualized by seeding micro-scale titanium dioxide $\left(\mathrm{TiO}_{2}\right)$ particles in the reactant gas and illuminating by laser (Wahl, 1977; Giling, 1982; Wang et al., 1986). However, the holography observation using the laser provided poor resolution of the lowly concentrated reactants. On the other hand, numerical simulations provide relatively better understanding of the fluid mechanics and have become very important to study the complex flow in CVD process.

Numerous researchers have been devoted to investigate the flow and heat transfer in CVD reactors. Some of them focused on the simulations of the horizontal CVD reactors (Moffat and Jensen, 1986; Fotiadis et al., 1990; Karki et al., 1993; Chiu, Richards et al., 2000; Chiu et al., 2001), while many other important studies have been conducted to the vertical configurations (Sugawara, 1972; Dimitrios et al., 1987). Among all the numerical analysis in CVD reactors, three major governing equations are considered - continuity, momentum, and energy conservations (Patankar, 1980). Generally, parabolic governing equations (Moffat and Jensen, 1986; Quazzani and Rosenberger, 1990) are utilized to predict the flow pattern in CVD reactors. However, extreme conditions, such as low Reynolds numbers and high density gradients, lead to reverse flow (Visser et al., 1989) which required elliptic governing equations for better predictions (Quazzani and Rosenberger, 1990; Karki et al., 1993).

Simulations of the CVD processes are very complicated because of huge amount of controlling variables (Raja et al., 2000), complicated analysis of the fluidic dynamics and the kinetics of the chemical reactions, and all the variable properties (Jensen et al., 1991) to be considered. Numerical models with constant properties (Eversteyn et al., 1970; Gebhart et al., 1988; Spall, 1996) and Boussinesq approximations (Gray and Giorgini, 1976; Gebhart et al., 1988) have been utilized to simplify the complex simulations. Wang et al.. (Wang et al., 2003) and Chiu et al. (Chiu, Jaluria et al., 2000) demonstrated that the constant-property models are acceptable for most practice but variable-property models give more accurate predictions for extreme operating conditions. The Buoyancy effect has been neglected when the ratio of Grashof number and square of Reynolds number is less than two (Quazzani et al., 1988; Chiu and Jaluria, 1997). The geometry of the reactor is also a factor in the fluid dynamics of the CVD process but it is negligible in a large aspect ratio (Chiu, Jaluria et al., 2000). The temperature distribution of the susceptor is approximated to be isothermal for some CVD configurations (Chiu and Jaluria, 1997).

Thorough comparisons between the experiment and simulation results are provided by Dimitrios et al. (Dimitrios et al., 1987) and Chiu et al. (Chiu et al., 2001). Comprehensive reviews on CVD reactor studies are given by Mahajan (Mahajan, 1996) and Jensen et al. (Jensen et al., 1991). Kee et al. (Kee et al., 1995; Raja et al., 2000) demonstrated that model simulations have much greater flexibility and versatility as compared to experimental counterparts. Experimental studies have also been carried out on the flow in channels for CVD applications (Jensen et al., 1991; Chiu, Richards et al., 2000; Chiu et al., 2001; Chiu et al., 2002).

\subsection{Optimization of the CVD Processes and Existence of the Design Uncertainties}

Simulation and optimization of CVD systems have been studied by many researchers (Mouche et al., 1995; Southwell et al., 1996; Chiu, 1999; Raja et al., 2000; Chiu et al., 2002; Ly and Tran, 2002; George, 2007; Lin, Jaluria et al., 2009). However, design uncertainties can found everywhere in the CVD process. Even if an optimal design is obtained from the optimization models, the irresistible uncertainties will cause unstable responses of the CVD process. For example, the compositions of the deposition species have errors of $15 \%$ (Mitchell et al., 1997). Several researchers have estimated the randomness of the operating parameters in the CVD process. The rate constant of the chemical reaction may have a wide variance; for example, $10^{13}$ to $10^{14} \mathrm{~cm}^{3} / \mathrm{mol}-\mathrm{s}$ (Goodwin, 1993). In this research work, the existence of the design uncertainties is considered at the design variables, the inlet velocity and the susceptor temperature of the CVD process.

The main contribution of this research includes

- The development of the performance responses in the CVD process.

- The parametric modeling of those responses in terms of the chosen design variables.

- The optimization formulations of the operating operations for the CVD process.

- The realization of the convergence problem in a traditional RBDO algorithm, the TRIA.

- The development of the MRIA to solve optimization problems with normally distributed design uncertainties.

- The development of the MRIA for the non-normally distributed random variables.

- The application of the MRIA on the RBDO problems of the CVD process.

The Fig. 3 schemes the systematic strategy to parametrically model the responses from the experiments or the simulations of the thermal systems and optimize the operating conditions with design uncertainties using the proposed RBDO algorithm.

At the beginning of the productions of any thermal systems, several trials of the experiments or the numerical simulations are necessary to determine the operating conditions and examine the system performances. A Response Surface Method (RSM) model helps the engineers or the designers recognize the behavior of the 
responses with respect to the design variables. Optimization problems are formulated in terms of the RSM models and are utilized to provide the operating conditions for higher productivity and quality of the productions. Due to the existence of the design uncertainties, the traditional deterministic optimization formulation is no longer reliable to generate safe designs because it may lead to a design with high risk of system failure. The development of the Reliability-Based Design Optimization (RBDO) algorithm evaluates the probabilities of the system failures and provides a more conservative design which reaches to the optimality as the failure probabilities are subject to some acceptable level. Finally, the productions of the thermal systems are executed based on the optimal design variables. If any design uncertainties are found in the experiments, the simulations, or the mass productions, the information of the uncertainties are fed back to the formulation of the RBDO problems and new optimal conditions can be generated by the proposed strategy.

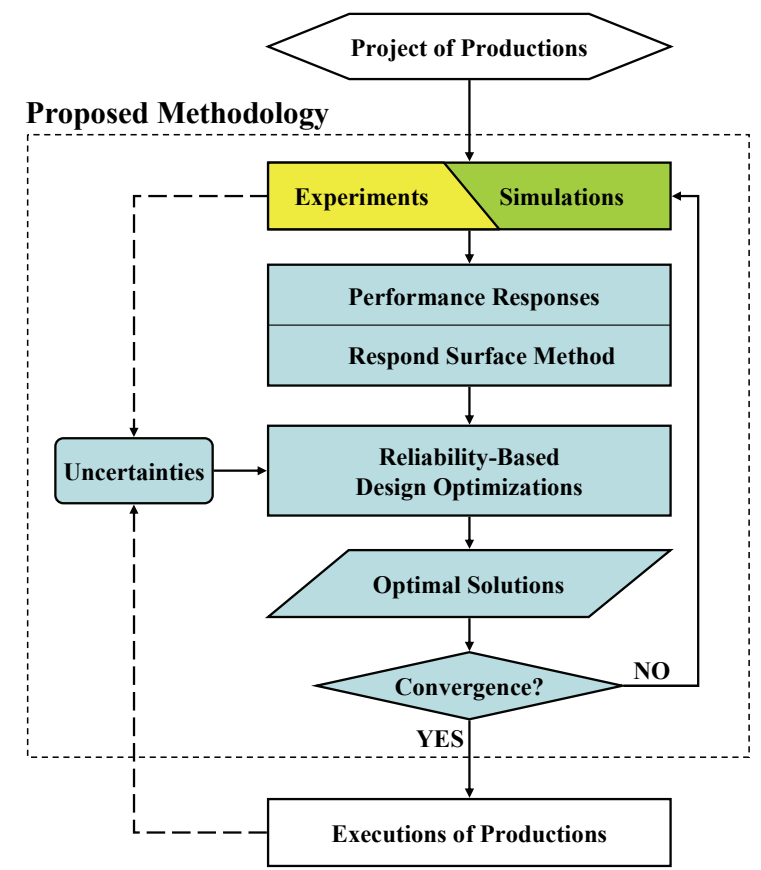

Fig. 3 Flowchart of the proposed systematic strategy.

\section{SIMULATION AND RESPONSES OF THE CVD PROCESS}

This session is directed at the simulation of the CVD process and the responses of to represent the productivity and the uniformity of the deposition. The effect of different operating conditions on the deposition rate and the film quality are identified from the numerical simulations performed using a commercial software, FLUENT. The simulation of the thin-film growth of silicon from the reactant of silane in a vertical impinging CVD reactor is discussed. The design operating conditions focus on the velocity of the inlet flow and the temperature of the susceptor. Four different responses are defined in terms of the design variables and measured from the simulated deposition profiles to represent the performances of the silicon deposition.

\subsection{Simulation of the CVD Process}

A schematic of the vertical impinging CVD reactor is shown in Fig. 4. The reaction gases are introduced at the top in a vertical impinging reactor, while the flow is assumed to be two-dimensional steady laminar flow (Chiu and Jaluria, 1999). The flow with a dilute precursor concentration of silane in hydrogen as the carrier gas deposits silicon onto the susceptor. Silane decomposes to silicon and hydrogen on the susceptor following a one-step reaction mechanism. The conservations of continuity, momentum, and energy are considered, shown by Eqs. (1), (2), and (3) respectively. Furthermore, the species conservation, shown by Eq. (4), is studied where there are three species, silane, silicon, and hydrogen, in the CVD chamber.

$$
\begin{aligned}
& \nabla \cdot \vec{v}=0 \\
& \vec{v} \cdot \nabla(\rho \vec{v})=\nabla \cdot(\mu \nabla \vec{v})+\vec{F}-\nabla p \\
& \nabla \cdot\left(\rho C_{P} \vec{v} T\right)=\nabla \cdot\left(k_{T} \nabla T\right)+\dot{Q} \\
& \nabla \cdot(\rho \vec{v} m)=\nabla \cdot(\rho D \nabla m)+R
\end{aligned}
$$

where $\vec{v}$ is the velocity of the flow, $T$ is the temperature, $m$ is the mass fraction of species, $\rho$ is the density, $\mu$ is the dynamic viscosity, $C_{P}$ is the specific heat, $k_{T}$ is the thermal diffusivity, $D$ is the mass diffusivity, $\vec{F}$ is the body force, $\nabla p$ is the pressure difference, $\dot{Q}$ is the heat source, and $R$ is the production rate of species.

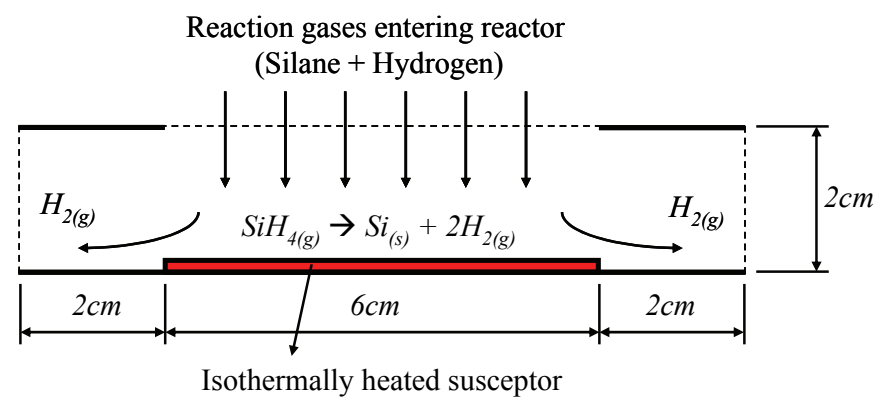

Fig. 4 Vertical impinging CVD reactor (George, 2007; Lin, 2010).

Simulations are carried out employing the commercial software FLUENT using the laminar finite-rate model, which computes the chemical source terms using Arrhenius expressions and ignoring the effects of turbulent fluctuations. (Lin, 2010) In the FLUENT model, both gas phase and wall surface reactions are considered, while the reaction rate is given by an Arrhenius expression, shown as follows

$\kappa=A T^{\alpha} \exp \left(-E_{a} / R_{g} T\right)$

where $\kappa$ is the rate constant, $A=0.334$ is the pre-exponential factor, $T$ is the temperature, $E_{a}=1 \times 10^{5}$ is the activation energy, $\alpha=0.5$ is the temperature exponent, and $R_{g}$ is universal gas constant. The material properties are given by the FLUENT database. The usage of FLUENT can accommodate different geometries and boundary conditions, providing more flexibility and saving more research time.

In the discretization scheme of the vertical CVD simulation, the power-law discretization scheme (Patankar, 1980) is used. For the solver, a efficient strategy (Lin et al., 2008) of using the Coupled and the PISO algorithms is used to approach the convergences of the governing equations of continuity, momentum, energy, and species transport simultaneously. The boundary conditions are shown in Fig. 5, where the walls are non-slip, impermeable, and thermally insulated at $300 \mathrm{~K}$. The susceptor is isothermally heated at temperature, $T$. The temperature of the reaction gases entering the reactor is $300 \mathrm{~K}$. The mass fraction of silane is kept at 0.1 because it ensures sufficient amounts of precursor gas in the mixture (Rebenne and Bhat, 1994). The inlet flow velocity, $V$, and susceptor temperature, $T$, contributes a lot in the flow and the deposition profile 
so they are considered as the design variables in this case study. The velocity and temperature bounds are taken from 0.1 to $1 \mathrm{~m} / \mathrm{s}$ and 400 to $1500 \mathrm{~K}$ respectively. Only half of the fluid domain is considered in the simulation due to the geometrical symmetry while four-node quad meshes are utilized - totally $320 \times 139$ nodes. Node density is chosen such that the solution is independent to the number of elements. For one typical simulation, it takes around 10 minutes to converge in an Intel ${ }^{\circledR}$ Pentium ${ }^{\circledR}$ M processor $2.0 \mathrm{GHz}$ with $2.0 \mathrm{GHz}$ of RAM.

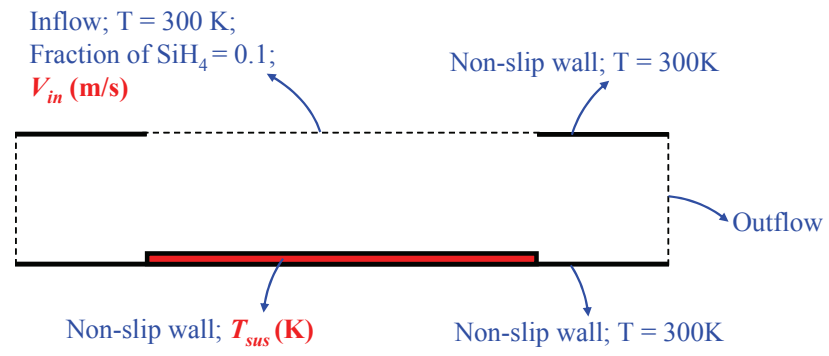

Fig. 5 Boundary conditions of the vertical CVD simulation.

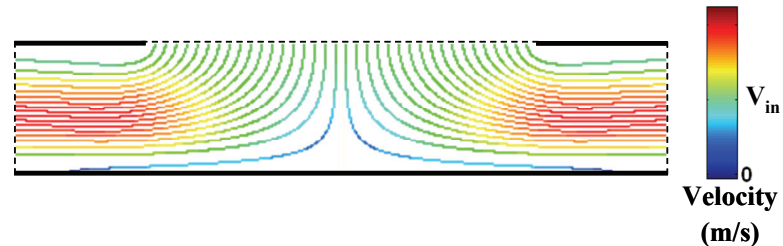

(a)

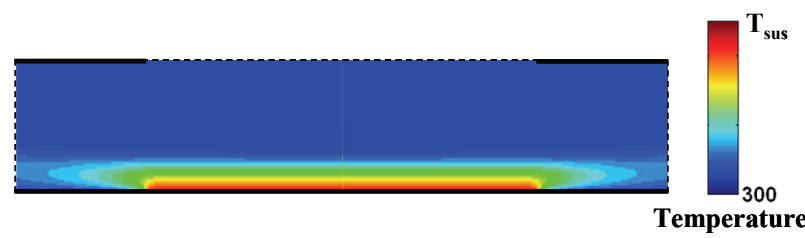

(K)

(b)

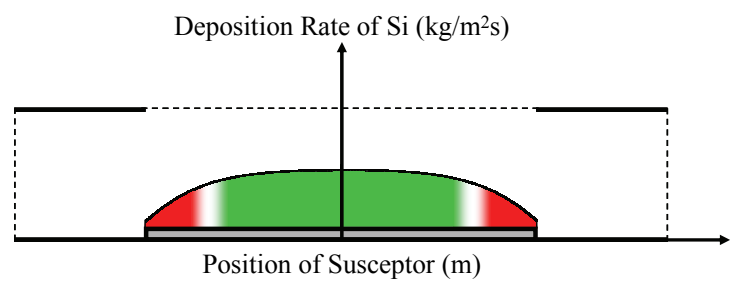

(c)

Fig. 6 Typical results of the vertical CVD simulation: (a) streamlines of the flow, (b) temperature distribution, and (c) scheme of silicon deposition profile (Lin, 2010).

The Fig. 6 (a) shows a typical numerical result of the streamlines in the CVD reactor where the inlet has uniformly distributed velocity, $V$, and the stagnation point at the center of the susceptor has zero velocity. The subfigure (b) illustrates the corresponding temperature distribution in the CVD chamber where the susceptor is isothermally heated at the temperature of $T$ and the walls remain room temperature following the boundary conditions. The subfigure (c) shows a scheme of the deposition profile of silicon while the red area has poor uniformity of the deposition rate. The red area is highlighted when its local slope of the deposition profile is too oblique; on the other hand, only the green area has local slopes very close to zero and the corresponding uniformity is acceptable uniformity.

\subsection{Validation}

To validate the correctness of the numerical simulations with the developed settings in FLUENT, the deposition rate of the silicon in a horizontal CVD reactor is compared with experimental and numerical results from other researchers (Eversteyn et al., 1970; Mahajan and Wei, 1991; Chiu and Jaluria, 2000; Yoo and Jaluria, 2002; George, 2007). The Fig. 7 illustrates the configuration of the horizontal CVD reactor and its dimensions. The reactant, the silane, is mixed in the hydrogen carrier flow, with the inlet velocity of $0.175 \mathrm{~m} / \mathrm{s}$. The partial pressure of the silane is $124.1 \mathrm{~Pa}$ under the atmospheric pressure, providing the information of the mass fraction of the silane. The operating temperature is $300 \mathrm{~K}$ and the susceptor is isothermally heated at $1323 \mathrm{~K}$. The material properties and the kinetics of the chemical reactions are given from (George, 2007) otherwise from the FLUENT database. The rest of the settings and the utilized solvers remain the same as the previous discussion.

Reaction gases entering reactor

(Silane + Hydrogen)

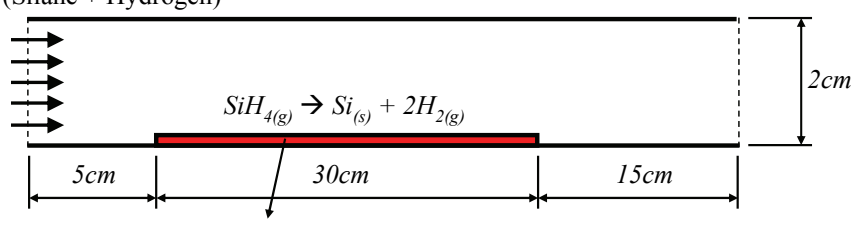

Isothermally heated susceptor

Fig. 7 Horizontal CVD reactor (George, 2007).

The results from the simulation of a horizontal CVD reactor using FLUENT are compared with the experimental results obtained by (Eversteyn et al., 1970) and the numerical results from (Mahajan and Wei, 1991; Chiu and Jaluria, 2000; Yoo and Jaluria, 2002), shown in Fig. 8. A detailed comparison between experimental and numerical results has been made and fairly good agreements has been found (Chiu, 1999). The numerical results using the settings described in the previous discussion is almost identical to George's simulation results (George, 2007) with the mass diffusivity in the FLUENT database, which is given by a polynomial equation of the temperature as follows:

$D=7.234 \times 10^{-8} T+4.569 \times 10^{-10} T^{2}-8.016 \times 10^{-14} T^{3}$

George (George, 2007) has also shown the numerical results are very close to the experimental results in (Eversteyn et al., 1970) if higher mass diffusivity is utilized. Eversteyn et al. (Eversteyn et al., 1970) utilized a power law to describe the mass diffusivity which is given by

$D=D_{0}(T / 300)^{\gamma}$

where $D_{0}$ is the pre-exponential factor, $\gamma$ is the temperature exponent, and the temperature, $T$, is normalized by the operating temperature, $300 \mathrm{~K}$. Considering the pre-exponential constant in (Yoo and Jaluria, 2002), $D_{0}=6.24 \times 10^{-5} \mathrm{~m}^{2} / \mathrm{s}$, and varying the temperature exponent from 1.7 to 2.0 as Eversteyn et al. (Eversteyn et al., 1970) suggested, different levels of the mass diffusivity of the silane are obtained and shown in Fig. 9. With the higher temperature exponent, the corresponding mass diffusivity increases as well as the growth rate of the silicon along susceptor in the horizontal CVD reactor increases, 
shown in Fig. 10. In this research work, the mass diffusivity is chosen from the FLUENT database.

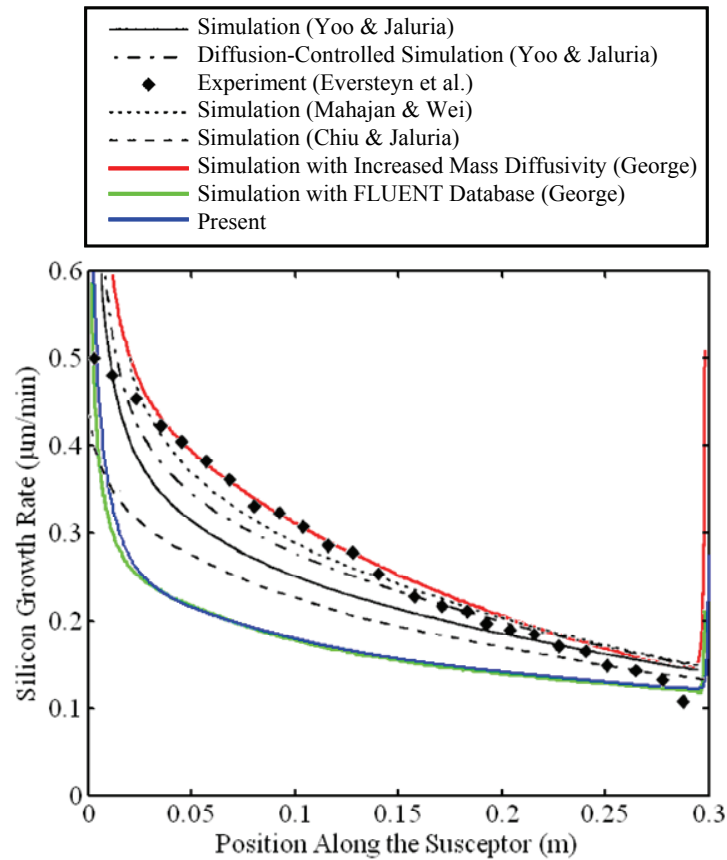

Fig. 8 Deposition rate of silicon in the horizontal CVD reactor compared with others (Eversteyn et al., 1970; Mahajan and Wei, 1991; Chiu and Jaluria, 2000; Yoo and Jaluria, 2002; George, 2007).

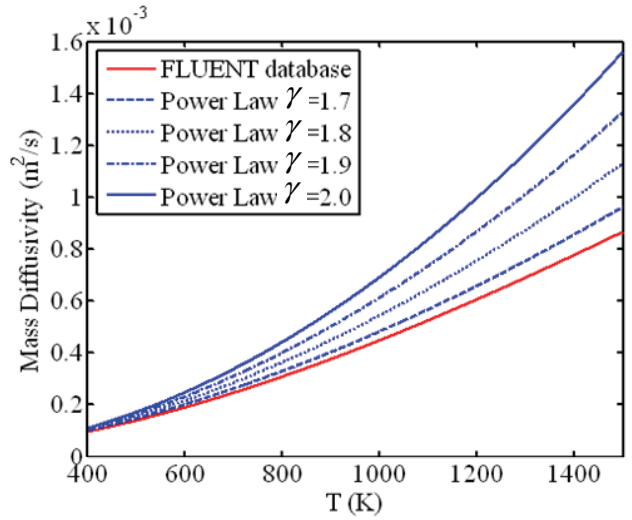

Fig. 9 Mass diffusivity of silane with different temperature exponent compared with FLUENT database.

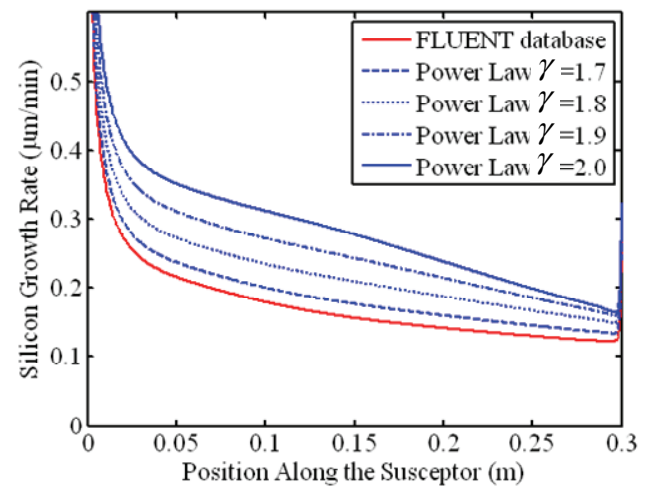

Fig. 10 Growth rate of silicon in the horizontal CVD reactor with different mass diffusivity.

\subsection{Function Formulations of the Responses in CVD Process}

Some quantitative measures are needed to justify the performance of the deposition profile to achieve higher deposition rate and better film quality on the susceptor during the CVD process. Four responses have been defined in (Lin et al., 2008), including the Percentage of the Working Area (PWA) and three mathematical functions - Mean of Deposition Rate (MDR), Root Mean Square (RMS) and Surface Kurtosis (KUR). The PWA is used because the quality of deposition close to the edge of the susceptor and the working area excludes all unusable areas. In the working area, the other three response functions represent the productivity and the uniformity of the silicon deposition.

Percentage of Working Area (PWA) is defined as the ratio of the Effective Working Area (EWA) and the total area in the deposition profile and it is given by

$\mathrm{PWA}=\frac{\mathrm{EWA}}{\text { Total Area }} \times 100 \%$

where the EWA is determined based on the local uniformity check and the continuity of the working area. A local area is considered as workable only when the local slope, $|S|$, is very close to zero or it is smaller than an uniformity threshold, $S_{U}$. In the study of the vertical CVD, the quantity of $S_{U}$ is chosen as a small value, 0.00055 . Normally, regions with higher slopes occur around the edges of the susceptor due to the dramatic temperature drop. However, there are some cases that the uniform areas are located far from the edge and result in discontinuous working areas. Those isolated uniform area cannot be utilized to produce micro-structures. Hence, the EWA is given as the largest set of the working area at the middle of the deposition profile excluding the rest of the isolated working areas. The rest of the responses should be measured within the EWA instead of the entire area of the susceptor because only the deposition performance in the working area is of concern and the non-working area will be dumped.

Mean of Deposition Rate (MDR) is measured inside the EWA as the key criteria to represent the performance of CVD process. It is given as

$\operatorname{MDR}=\frac{1}{Q} \sum_{q=1}^{Q} D_{q}$

where $Q$ is the number of uniformly distributed sampling nodes within the EWA, and $D_{q}$ is the deposition rate at the sampling node.

The higher MDR represents better CVD productivity. A deposition profile with a larger PWA along with higher MDR is very desirable; however, these two objectives sometimes conflict with each other. In the later session, two different kinds of the optimization formulations will be studied - one maximizes the PWA and the other one maximizes the MDR.

Root Mean Square (RMS) and Surface Kurtosis (KUR): The PWA only provides a local measure for uniformity, therefore it is essential to determine the global uniformity mathematically and represent the global physical behaviors of the deposition profile within the EWA. Two statistical measures, the Root Mean Square (RMS) and the Surface Kurtosis (KUR), are utilized to quantify the global uniformity.

The Root Mean Square (RMS) is used to measure the magnitude of the varying deposition rate, shown as 


$$
\mathrm{RMS}=\sqrt{\frac{1}{Q} \sum_{q=1}^{Q}\left(D_{q}-\mathrm{MDR}\right)^{2}}
$$

which is the standard derivation of the deposition rate of all sampling nodes within the EWA. A deposition profile with large RMS represents un-even uniformity of the thin-film formation even all regions are considered as workable from the PWA point of view. Therefore, the RMS is an important global indicator for the film quality, which is complementary to the local indicator, PWA.

The KUR is the forth statistical moment of the deposition profile and it mathematically implies the existences of the sharper peaks in the deposition profile which cannot be recognized by the RMS. It is measured by

$$
\mathrm{KUR}=\frac{1}{Q(\mathrm{RMS})^{4}} \sum_{q=1}^{Q}\left(D_{q}-\mathrm{MDR}\right)^{4}
$$

where the terms of MDR and the RMS are defined in the Eqs. (9) and (10) respectively. The deposition profile with a higher KUR has greater peakedness or higher variance due to infrequent extreme deviations. A silicon deposition with allowable KUR is desired. Also, the skewness is not considered because the configuration of the vertical CVD reactor is symmetric about the centerline of the inlet flow and the skewness is always zero. For other geometrical configurations, the skewness is also an important factor to study the global uniformity and symmetry.

The percentage of the EWA in the susceptor has been defined to quantify the production yield of the CVD process. The MDR is measured within the EWA to represent the productivity, while the RMS and the KUR are utilized as the indicators of the uniformity inside the EWA. Several samples of the CVD simulations under different operating conditions have been utilized to show the importance of four responses in the quantifications of the productivity and the uniformity of the CVD process. Both RMS and KUR are global measures for the quality of deposition uniformity whereas the PWA and MDR are direct local measures. These four functions are greatly influenced by the inlet velocity and the susceptor temperature. However, the true forms of these functions are not available. Therefore, we will establish response surface models of these functions with respect to the design variables.

\subsection{Parametric Modeling Using Response Surface Method (RSM)}

The best way to represent the behavior of the responses in the CVD processes and model them in terms of numerical functions with respect to the design variables is to use the technique of curve fitting, also known as metamodeling or Response Surface Method (RSM). RSM provides a parametric equation in terms of the design variables and some coefficients to be determined by substituting the experiment / simulation data into the parametric model. Instead of using the common RSM tool, Polynomial Response Surface (PRS), the Radial Basis Function (RBF) is used to model the deposition rate and the uniformity of the deposition profile. These obtained parametric models of the four responses will be validated before being utilized to formulate the optimization problems of CVD process.

Response Surface Method can be divided into two different types (Jaluria, 1998):

- $\quad$ Exact fitting.

- Best fitting.

The exact fitting is the technique that generates a smooth curve that passes through all the data points. It typically is a function with $M$ parameters to be determined by the known information from $M$ data points. Therefore, it is very accurate and useful for small amount of data. On the other hand, the best fitting does not necessarily pass through any of the data points but provides a best prediction of the behavior of the responses. It is very useful when the amount of data points is very large (i.e. There are only $K$ parameters to be determined for $M$ sampling points while $K<M$.) or the obtained responses are not accurate enough. Several methods have been developed to achieve either exact or best fitting with single variable, two variables, or multiple variables. Table 4 lists different kinds the RSM methods and their characteristics.

Table 4 Different RSM methods (Jaluria, 1998; Van Beers and Kleijnen, 2003; Van Beers and Kleijnen, 2004; Mortenson,

\begin{tabular}{|c|c|c|c|}
\hline Methods & Available Dimensions & $\begin{array}{l}\text { Exact } \\
\text { Fitting }\end{array}$ & $\begin{array}{c}\text { Best } \\
\text { Fitting }\end{array}$ \\
\hline $\begin{array}{l}\text { Polynomial } \\
\text { fitting }\end{array}$ & Multiple variables & O & $\bigcirc$ \\
\hline $\begin{array}{l}\text { Hermite curve } \\
\text { / surface }\end{array}$ & Curve for $1 \mathrm{D}$; surface for $2 \mathrm{D}$ & & \\
\hline $\begin{array}{c}\text { Bezier curve / } \\
\text { surface }\end{array}$ & Curve for $1 \mathrm{D}$; surface for $2 \mathrm{D}$ & & ○ \\
\hline $\begin{array}{l}\text { B-spline curve } \\
\text { / surface }\end{array}$ & Curve for $1 \mathrm{D}$; surface for $2 \mathrm{D}$ & & $\bigcirc$ \\
\hline Kriging & Multiple variables & $\bigcirc$ & $\bigcirc$ \\
\hline $\begin{array}{l}\text { Radial basis } \\
\text { function }\end{array}$ & Multiple variables & $\bigcirc$ & $\Omega$ \\
\hline $\begin{array}{c}\text { Thin plate } \\
\text { spline }\end{array}$ & Multiple variables & $\bigcirc$ & $\bigcirc$ \\
\hline
\end{tabular}
2006).

Polynomial Response Surface (PRS) is the most common and simple technique to interpolate or extrapolate the obtained responses with $M$ sampling points. For small-scale models, a regression formulation is given as follows:

$F(\boldsymbol{x}) \cong \boldsymbol{w} \cdot \boldsymbol{B}(\boldsymbol{x})$

where $\boldsymbol{w}$ is a vector of the $K$ regression coefficients to be determined and $\boldsymbol{B}(\boldsymbol{x})$ is a linear combination of the modeling monomials, shown in Table 5.

Table 5 Typical coefficients for PRS.

\begin{tabular}{|c|c|c|}
\hline Different PRS & $K$ & $\boldsymbol{B}(\boldsymbol{x})$ \\
\hline Linear regression & 2 & {$\left[\begin{array}{ll}1 & x_{1}\end{array}\right]^{T}$} \\
\hline Planar regression & 3 & {$\left[\begin{array}{lll}1 & x_{1} & x_{2}\end{array}\right]^{T}$} \\
\hline $\begin{array}{c}\text { Coupling 2-D fitting } \\
\text { Independent 2-D quadratic } \\
\text { fitting }\end{array}$ & 4 & {$\left[\begin{array}{llll}1 & x_{1} & x_{2} & x_{1} x_{2}\end{array}\right]^{T}$} \\
\hline $\begin{array}{c}\text { Coupling 2-D quadratic } \\
\text { fitting }\end{array}$ & 6 & {$\left[\begin{array}{lllll}1 & x_{1} & x_{2} & x_{1}^{2} & x_{2}^{2}\end{array}\right]^{T}$} \\
\hline
\end{tabular}

For exact fitting, $K=M$ and the coefficients, $\boldsymbol{w}$, can be obtained by the following equation:

$\boldsymbol{w}=\boldsymbol{A}^{-1}\left(\boldsymbol{x}^{S}\right) \cdot \boldsymbol{F}^{S}\left(\boldsymbol{x}^{S}\right)$

where $\boldsymbol{F}^{S}\left(\boldsymbol{x}^{S}\right)$ is a $K \times 1$ vector of the responses in terms of the $K$ sampling points and $\boldsymbol{A}\left(\boldsymbol{x}^{S}\right)$ is a $K \times K$ matrix whose $s^{\text {th }}$ row 
is $\boldsymbol{B}^{T}\left(\boldsymbol{x}_{s}^{S}\right)$. For best fitting, $K<M$ and $\boldsymbol{F}^{S}\left(\boldsymbol{x}^{S}\right)$ is a $M \times 1$ vector and $\boldsymbol{A}\left(\boldsymbol{x}^{S}\right)$ is a $M \times K$ matrix. Instead of using Eq. (13), the coefficients of $\boldsymbol{w}$ need to be obtained by Least Square Approximation (Myers et al., 2009) shown as follows:

$$
\boldsymbol{w}=\left[\boldsymbol{A}^{T}\left(\boldsymbol{x}^{S}\right) \cdot \boldsymbol{A}\left(\boldsymbol{x}^{S}\right)\right]^{-1} \cdot \boldsymbol{A}^{T}\left(\boldsymbol{x}^{S}\right) \cdot \boldsymbol{F}^{S}\left(\boldsymbol{x}^{S}\right)
$$

Geometric Modeling Curves and Surfaces: There are several famous geometric modeling methods that can be utilized for the purpose of RSM (Mortenson, 2006). Those include Hermite, Bezier, and B-Spline curves for single-variable RSM and Hermite, Bezier, and B-Spline surfaces for two-variable RSM. A Hermite cubic curve contain multiple continuously connected cubic curves which are parameterized by polynomial functions in terms of four sampling points. The parametric equation is a single-variable polynomial fitting function in Eq. (12) with $\boldsymbol{B}(\boldsymbol{x})=\left[\begin{array}{llll}1 & x_{1} & x_{1}^{2} & x_{1}^{3}\end{array}\right]^{T}$. The advantage of using a known parametric function like Hermite curve is that the coefficients, $\boldsymbol{w}$, are given already and no further calculation is needed for the determination of $\boldsymbol{w}$. However, it is generally used for exact fitting. Bezier and B-spline curves are utilized for best fitting of the responses with different set of coefficients, $\boldsymbol{w}$. More details are included in (Mortenson, 2006).

Kriging is a general method to predict the responses from the experiment or simulation data with minimum error variance estimation (Lebensztajn et al., 2004). It is constructed by an inner product of a vector of coefficients, $\boldsymbol{w}$, and a covariance vector, $\boldsymbol{C}_{x}\left(\boldsymbol{x}, \boldsymbol{x}^{S}\right)$, shown as follows:

$F(\boldsymbol{x}) \cong \boldsymbol{w} \cdot \boldsymbol{C}_{x}\left(\boldsymbol{x}, \boldsymbol{x}^{S}\right)$

Table 6 Common covariance functions for different Kriging techniques.

\begin{tabular}{|c|c|}
\hline Different Kriging Techniques & $\operatorname{Cov}\left(\boldsymbol{x}_{r}^{S}, \boldsymbol{x}_{s}^{S}\right)$ \\
\hline $\begin{array}{c}\text { Gaussian covariance function } \\
\text { (Simpson et al., 2001; Van Beers } \\
\text { and Kleijnen, 2004) }\end{array}$ & $\left(\sigma^{S}\right)^{2} \sum_{h} \exp \left[-\theta_{h}\left\|x_{h, r}^{S}, x_{h, s}^{S}\right\|^{2}\right]$ \\
\hline $\begin{array}{c}\text { Exponential covariance function } \\
\text { (Van Beers and Kleijnen, 2004) }\end{array}$ & $\left(\sigma^{S}\right)^{2} \sum_{h} \exp \left[-\theta_{h}\left\|x_{h, r}^{S}, x_{h, s}^{S}\right\|\right]$ \\
\hline $\begin{array}{c}\text { Product-form covariance function } \\
\text { (Van Beers and Kleijnen, 2004; } \\
\text { Den Hertog et al., 2006) }\end{array}$ & $\left(\sigma^{S}\right)^{2} \prod_{h} \exp \left[-\theta_{h}\left\|x_{h, r}^{S}, x_{h, s}^{S}\right\|\right] *$ \\
\hline $\begin{array}{c}\text { Radial basis function (Giesl, } \\
\text { 2007) }\end{array}$ & $\left\|\boldsymbol{x}_{r}^{S}, \boldsymbol{x}_{s}^{S}\right\|$ \\
\hline $\begin{array}{c}\text { Pseudo-cubic spline (Duchon, } \\
\text { 1977) }\end{array}$ & $\left\|\boldsymbol{x}_{r}^{S}, \boldsymbol{x}_{s}^{S}\right\|^{3}$ \\
\hline $\begin{array}{c}\text { Weighted distance function (Van } \\
\text { Beers and Kleijnen, 2003; Jeong } \\
\text { et al., 2004) }\end{array}$ & $\sum_{h} \theta_{h}\left\|x_{h, r}^{S}, x_{h, s}^{S}\right\|^{\lambda_{h}} \S$ \\
\hline $\begin{array}{c}\text { Thin plate spline (Lebensztajn et } \\
\text { al., 2004) }\end{array}$ & $\left\|\boldsymbol{x}_{r}^{S}, \boldsymbol{x}_{s}^{S}\right\|^{2} \log \left\|\boldsymbol{x}_{r}^{S}, \boldsymbol{x}_{s}^{S}\right\|$ \\
\hline
\end{tabular}

$\dagger \sigma^{S}$ stands for the known standard deviation of the responses and $\theta_{h}$ is the unknown correlation parameter to be determined by maximizing the likelihood estimates.

$\$ h$ denotes the index for the important sampling points and there exists more than one important sampling points.

$\S \lambda_{h}$ controls the smoothness of the distance function. where the covariance vector contains $M \times 1$ components of the covariance functions and is given by

$\boldsymbol{C}_{x}\left(\boldsymbol{x}, \boldsymbol{x}^{S}\right)=\sum_{s=1}^{M} C_{x, s}\left(\boldsymbol{x}, \boldsymbol{x}_{s}^{S}\right) \boldsymbol{e}_{s}=\sum_{s=1}^{M} \operatorname{Cov}\left(\boldsymbol{x}, \boldsymbol{x}_{s}^{S}\right) \boldsymbol{e}_{s}$

and $\boldsymbol{e}_{s}$ is the $s^{\text {th }}$ normal basis. The coefficients of $\boldsymbol{w}$ can be obtained by

$\boldsymbol{w}=\boldsymbol{C}^{-1}\left(\boldsymbol{x}^{S}\right) \cdot \boldsymbol{F}^{S}\left(\boldsymbol{x}^{S}\right)$

where

$\boldsymbol{C}\left(\boldsymbol{x}^{S}\right)=\sum_{r=1}^{M} \sum_{s=1}^{M} \operatorname{Cov}\left(\boldsymbol{x}_{r}^{S}, \boldsymbol{x}_{s}^{S}\right) \boldsymbol{e}_{r} \boldsymbol{e}_{s}$

is a $M \times M \quad$ symmetric matrix with zero diagonal terms and non zero off diagonal terms of covariance functions, $\operatorname{Cov}\left(\boldsymbol{x}_{r}^{S}, \boldsymbol{x}_{s}^{S}\right)$. Many different covariance functions have been utilized for the predictions of the response surfaces and they are listed in The RBF has no approximation error at all sampling points and is expected to provide better approximations of the responses with non-linear behaviors than the simple PRS.. Besides, other available covariance functions are constructed in a class of polynomial functions in terms of the basis function, $\left\|\boldsymbol{x}_{r}^{S}, \boldsymbol{x}_{s}^{S}\right\|$, shown in (Wendland, 1995). This kind of Kriging technique is utilized for exact fitting. On the other hand, a linear combination of the polynomial fitting function in Eq. (12) and the Kriging function in Eq. (15) makes best fitting possible for Kriging (Simpson et al., 2001). The polynomial term provides the global shape of the response surface, while the Kriging term provides local predictions of the responses.

\subsection{Parametric Modeling of the CVD Responses}

Radial Basis Function (RBF) (Giesl, 2007) is a special kind of Kriging, where the covariance function is the Euclidean distance between two corresponding vectors, namely,

$\operatorname{Cov}\left(\boldsymbol{x}_{r}^{S}, \boldsymbol{x}_{s}^{S}\right)=\left\|\boldsymbol{x}_{r}^{S}, \boldsymbol{x}_{s}^{S}\right\|$

It is preferred because the approximation of PRS may not be very accurate if the response is highly nonlinear. The RBF approximates the response, $F$, as a weighted summation of covariance functions:

$F \cong \sum_{s=1}^{M} w_{s} \operatorname{Cov}\left(\boldsymbol{x}, \boldsymbol{x}_{s}^{S}\right)$

where $w_{s}$ is the weighting factor to be determined. Two CVD operation parameters are studied, inlet velocity, $V$ and susceptor temperature, $T$. The four functions described previously are approximated using the RBF in the design domain which contains $M$ sampling points, $\boldsymbol{x}_{s}^{S}=\left[\begin{array}{ll}V_{s}^{S} & T_{s}^{S}\end{array}\right]^{T}$ for $s=1,2, \ldots, M$. Therefore, the Eq. (19) is given as

$\operatorname{Cov}\left(\boldsymbol{x}, \boldsymbol{x}_{s}^{S}\right)=\sqrt{\left(\frac{V-V_{s}^{S}}{V_{m}}\right)^{2}+\left(\frac{T-T_{s}^{S}}{T_{m}}\right)^{2}}$

where $V$ varies from 0.1 to $1 \mathrm{~m} / \mathrm{s}$ and $T$ varies from 400 to $1500 \mathrm{~K}$. The velocity and temperature differences are normalized by 
$V_{m}=1.0 \mathrm{~m} / \mathrm{s}$ and $T_{m}=1500 \mathrm{~K}$. The RBF has no approximation error at all sampling points and is expected to provide better approximations of the responses with non-linear behaviors than the simple PRS.

Model Validation: The RSM needs to be validated before the formulation of the optimization problems. Lin et al. (Lin, Jaluria et al., 2009) studied the RBFs with three different kinds of sampling sets uniformly distributed 9-point, 13-point, and 25-point designs. In this case study, uniformly distributed sampling is considered but another famous sampling method is the Latin Hypercube Sampling (Stein, 1987). A 437-point response was considered as a benchmark of the true responses. The detailed information of the 437 sampling points can be found in (Lin, 2010). The difference between the studying sample set and the 437-point response is quantified by an error function give as

$$
e r r=\sqrt{\frac{1}{437} \sum_{p=1}^{437}\left(F_{p}-G_{p}\right)^{2}}
$$

where $F_{p}$ and $G_{p}$ denote the nodal values of the approximate function and the simulation respectively. The results showed the 25point design has low errors (less than $4 \%$ ) while a 25 -point polynomial response surface has less accuracy due to the nonlinearity of the responses. For simplicity, the following sessions will use the 25-point RBF.

The Fig. 11 demonstrates the 25-point RBFs of the PWA, MDR, RMS, and KUR of the silicon deposition profiles. Due to the occurrence of the isolated working areas at high inlet velocity and low susceptor temperature, a sudden drop of PWA is found around that region. This sudden decrease of the PWA causes non-smooth behaviors during the transition. Unlike the models of PWA, the MDR behavior is very smooth because the MDR is an averaged value in the EWA and any non-linear behavior is blended into the entire function.

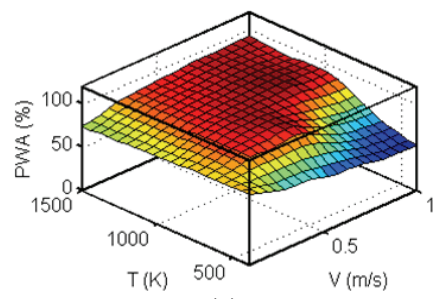

(a)

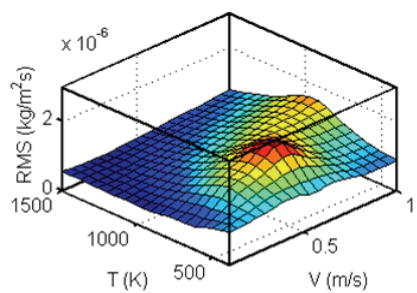

(c)

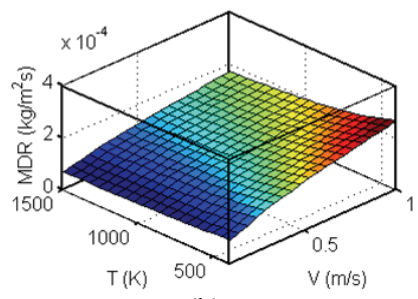

(b)

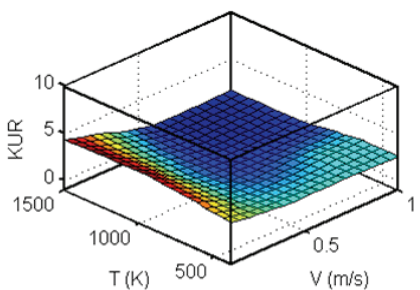

(d)
Fig. 11 25-Point RBFs of (a) PWA, (b) MDR, (c) RMS, and (d) KUR.

The proposed RBF can be utilized to parametrically model the responses in any other thermal systems. It is especially useful when the responses are highly nonlinear; otherwise, the polynomial response surface is less complicated and good enough for less nonlinear functions. In the next session, those response surface modeled are to be utilized to formulate optimization problems for enhancing the productivity and the quality of the silicon deposition. Besides obtaining a best set of the operating parameters of the CVD processes, the system reliability at the optimal setting is also an important study.
In practice, the optimal setting is only useful only when the corresponding system reliability is acceptable.

\section{OPTIMIZATION OF THE CVD PROCESS}

The response surface models are used to formulate the optimization problems of the CVD process while better operating conditions are expected to be found to improve the performance of the CVD process in this session. The objective and constraint functions are formulated by the RBF models of the CVD responses. For higher productivities, one of the objective functions is to maximize the PWA while another one is to maximize the MDR. On the consideration of the uniformity of the deposition, the RMS and the KUR are subject to certain quantitative levels. Both constraints of the deposition uniformity are very significant to the locations of the optimal operating conditions. Furthermore, the system reliability with respect to the optimal solution is of another significant concern. Without the consideration of the randomness of the system, there exists high risk of system failure when operating the system with the optimal parameters. To overcome this problem, a systematic method is desired to provide more conservative designs than the traditional ones.

\subsection{Problem Formulations}

In a general CVD process, the PWA and the MDR should be maximized in order to obtain the highest productivity, and two global uniformity factors, the RMS and the KUR are required to satisfy some desirable guidelines. Therefore, two optimization formulations (Lin et al., 2008) are proposed. The first one is to maximize the PWA with uniformity constraints on the RMS and the KUR while the deposition rate is subjected to a minimum level. The second one is to maximize the MDR with the same uniformity constraints while the PWA must be larger than an acceptable level. The detailed information about the optimal solutions is given later. Other possible formulations are shown in (Lin, 2010).

Example 1a - Maximizing the PWA Subject to Constraint of Deposition Rate: The first formulation is to maximize the working area on the susceptor subject to the constraints of the global uniformity factors described previously and an additional constraint on the deposition rate. It is expressed as follows:

$\begin{array}{ll}\underset{V, T}{\operatorname{Max}} & \text { PWA } \\ \text { s.t. } & {\text { RMS } \leq \mathrm{RMS}_{\mathrm{U}}} \\ & \text { KUR } \leq \mathrm{KUR}_{\mathrm{U}} \\ & \mathrm{MDR}_{\mathrm{L}} \leq \mathrm{MDR} \\ & V_{L} \leq V \leq V_{U} \\ & T_{L} \leq T \leq T_{U}\end{array}$

where the limit states of the constraints are taken as $\mathrm{RMS}_{\mathrm{U}}=1.35 \times 10^{-6}, \mathrm{KUR}_{\mathrm{U}}=2.62$, and $\mathrm{MDR}_{\mathrm{L}}=1.5 \times 10^{-4} \mathrm{~kg} / \mathrm{m}^{2} \mathrm{~s}$. The chosen bounds are $V_{L}=0.2 \mathrm{~m} / \mathrm{s}, V_{U}=0.9 \mathrm{~m} / \mathrm{s}, T_{L}=400 \mathrm{~K}$, and $T_{U}=1400 \mathrm{~K}$ because the interior of the responses surface model has better approximation than its edges (Lin, 2010). The Fig. 12 shows the feasible regions in white background color and the infeasible ones in three different colors, where red color is used for the first constraint, purple area indicates the infeasible domain of the second constraint, and the blue one is for the third MDR constraint. Multiple starting points are used to find the global optimal due to the disjoint feasible regions. The optimal solution, listed in Table 7, has been verified by the FLUENT simulation as well as the 437 samplings.

Example 2a - Maximizing the MDR Subject to Constraint of Working Area: The other formulation maximizes the MDR subject 
to the constraints of the uniformity factors and an additional constraint of the PWA, which is written as follows:

$$
\begin{array}{ll}
\underset{V, T}{\operatorname{Max}} & \text { MDR } \\
\text { s.t. } & {\text { RMS } \leq \mathrm{RMS}_{\mathrm{U}}} \\
& \mathrm{KUR} \leq \mathrm{KUR}_{\mathrm{U}} \\
& \text { PWA }_{\mathrm{L}} \leq \mathrm{PWA} \\
& V_{L} \leq V \leq V_{U} \\
& T_{L} \leq T \leq T_{U}
\end{array}
$$

where the PWA is desired to exceed the lower bound of $\mathrm{PWA}_{\mathrm{L}}=85 \%$. The Fig. 13 demonstrates the feasible region and the optimal design. Multiple starting points are also used to guarantee the solution is the global optimal. The optimal solution is listed in Table 7 and verified by the FLUENT simulation and the 437-point samplings.
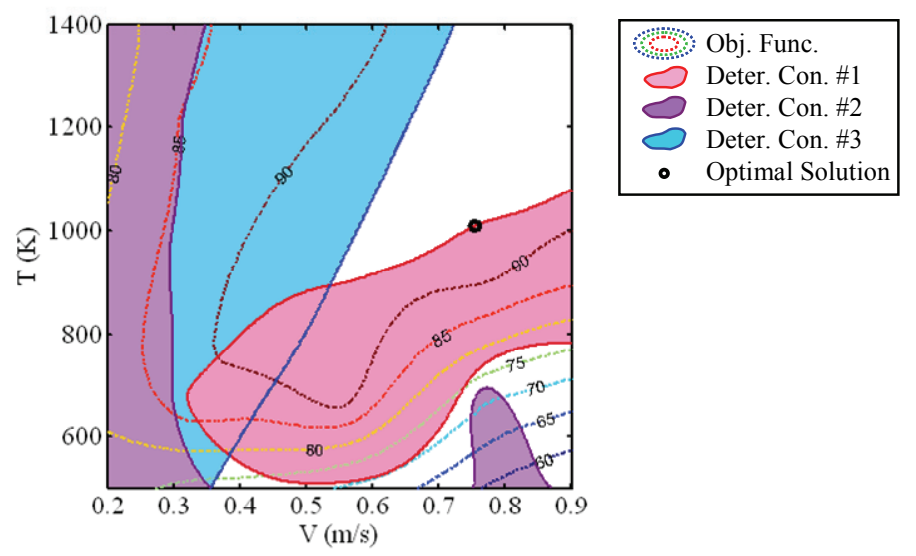

Fig. 12 Optimal solution of example 1a.

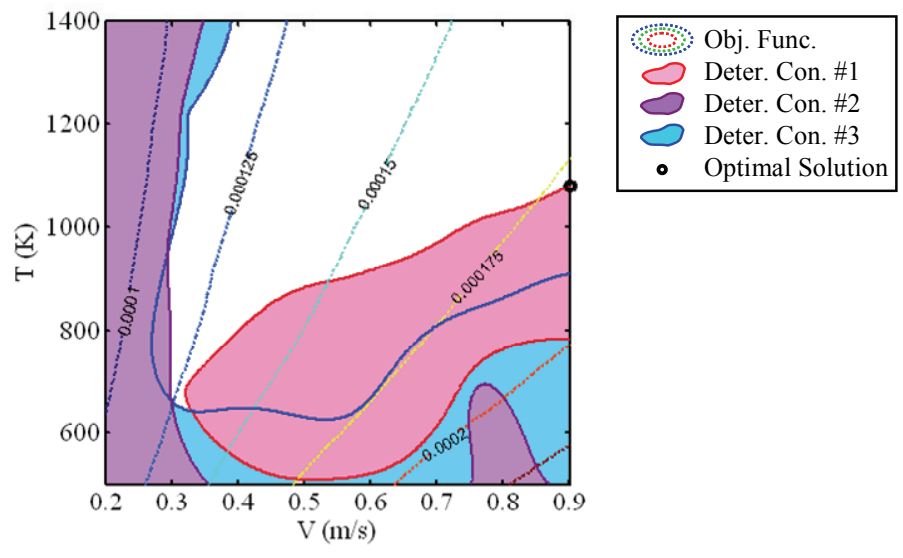

Fig. 13 Optimal solution of example 2a.

Table 7 Monte Carlo Simulations at the optimal solutions for examples 1 and 2.

\begin{tabular}{|c|c|c|c|c|c|}
\hline \multirow{2}{*}{ Ex. } & $\begin{array}{c}\text { Optimal } \\
\text { Solution }\end{array}$ & $\begin{array}{c}\text { Distribution } \\
\text { of Uncertainty }\end{array}$ & $M C S_{1}$ & $M C S_{2}$ & $M C S_{3}$ \\
\hline \multirow{2}{*}{ 1a } & \multirow{2}{*}{$(0.75,1009)$} & Normal & $42.90 \%$ & $0 \%$ & $0 \%$ \\
\cline { 3 - 6 } & & Lognormal & $43.02 \%$ & $0 \%$ & $0 \%$ \\
\hline \multirow{2}{*}{ 2a } & $(0.90,1079)$ & Normal & $50.10 \%$ & $0 \%$ & $0 \%$ \\
\cline { 3 - 6 } & & Lognormal & $50.33 \%$ & $0 \%$ & $0 \%$ \\
\hline
\end{tabular}

The deposition process of silicon from silane in a vertical impinging CVD reactor has been modeled and studied. Two quality factors, the Percentage of Working Area and the Mean of Deposition Rate were defined and two global uniformity factors, the Root Mean Square and the Surface Kurtosis, were modeled. The responses were approximated using the Radial Basis Function with respect to the two operation parameters - the inlet velocity and the susceptor temperature. Using the RBF models, two optimization formulations were proposed to maximize the productivity while maintaining a specific minimum level of the global uniformity factors. The obtained optimal solutions of the design variables have been verified by the simulations at the optimal points, and the solutions were found to be all feasible. Good agreements have been found between the optimization with 25 point models and 437 samplings. Therefore, not only the 25 point RBF models have fairly good approximations of the responses of the CVD process, but they are capable of providing correct optimal solutions. It is expected that the same methodology can be used in the deposition of many other materials such as titanium nitride (TiN), gallium nitride $(\mathrm{GaN})$, silicon carbide $(\mathrm{SiC})$, etc.

Optimization with Design Uncertainties: If the design uncertainties exist in the design variables, they can be mathematically described by some statistical distributions. Theoretically, the design with $100 \%$ system reliability does not exist. That is to say, the probability of system failure is non-zero; therefore, an optimal solution with zero failure probabilities does not exist. A lower bound is needed for these probabilistic constraints where the failure probabilities are subject to some allowable level. For most engineering practice, the allowable failure probability is less than $1 \%$.

Suppose that the inlet velocity and the susceptor temperature are normally distributed random variables with the standard deviations of $0.02 \mathrm{~m} / \mathrm{s}$ and $20 \mathrm{~K}$ respectively. The mean of the distributions are located at the optimal solutions obtained from the previous optimization problems. Monte Carlo Simulations show that the optimal designs in Examples 1a and 2a have high risks of system failures. The Table 7 shows the failure probabilities of each constraint at the optimal solutions with normally distributed random variables. For Example 1a and 2a, the optimal designs have high probabilities of $42.9 \%$ and $50.1 \%$ respectively to violations of the first constraints. Without the considerations of the design uncertainties, the thermal systems have high risks of the constraint violations resulting in massive defective productions. Additionally, the MCS results of the failure probabilities with lognormally distributed operating conditions are shown in Table 7. Unacceptable results are also found as some of the constraints have failure probabilities far larger than $1 \%$.

The optimal design of the thermal system becomes unreliable if the uncertainties exist in the design. As the traditional optimization algorithm pushes the design variables to the optimality, they are often on the limit state of the performance constraints or very close to them. The existence of the design uncertainties gives high probabilities of that the constraint limits are violated at the optimal solutions. Thus, an improved strategy is highly necessary to optimize the thermal system with design uncertainties.

\subsection{Reliability-Based Design Optimization (RBDO)}

Instead of using the traditional optimization formulations and neglecting the design uncertainties, many Reliability-Based Design Optimization (RBDO) algorithms have been developed to formulate probabilistic constraints while the probability of system failures is subjected to an acceptable level. Under the framework of RBDO, a more conservative design is expected to be determined based on the optimality and the feasibilities of the probabilistic constraints.

General RBDO Formulation: Consider the random design variables, $\boldsymbol{X}$, where the $j^{\text {th }}$ random design variable, $X_{j}$, has an 
expected value of $d_{j}$ and a standard deviation of $\sigma_{j}$. The general probabilistic design optimization is formulated as follows:

$$
\begin{array}{ll}
\underset{\boldsymbol{d}}{\operatorname{Min}} & z(\boldsymbol{d}) \\
\text { s.t. } & P\left[g_{i}(\boldsymbol{X})>0\right] \leq P_{f, i} \quad i=1 \ldots n \\
& \boldsymbol{d}^{L} \leq \boldsymbol{d} \leq \boldsymbol{d}^{U}
\end{array}
$$

where $P_{f, i}$ is the $i^{\text {th }}$ allowable probability of the system failure. Mathematically, probability of the system failure, $P\left[g_{i}(\boldsymbol{X})>0\right]$, can be calculated by an integral of its Joint Probability Density Function (JPDF), $f_{i}(\boldsymbol{x})$, within the infeasible domain and it is given as

$$
P\left[g_{i}(\boldsymbol{X})>0\right]=\int_{g_{i}(\boldsymbol{X})>0} \ldots \int f_{i}(\boldsymbol{x}) d x_{1} \cdots d x_{N}
$$

However, it is very computationally expensive to evaluate the JPDF and the failure probability in Eq. (26). Instead, two famous RBDO algorithms, Reliability Index Approach (RIA) (Hasofer and Lind, 1974; Lin, Gea et al., 2009) and Performance Measure Approach (PMA) (Tu et al., 1999), have been developed to generate linear approximate probabilistic constraints. They are widely utilized to solve RBDO problems because the approximate constraints have deterministic forms and are solvable by any general optimization algorithms.

Reliability Index Approach (RIA): Hasofer and Lind (Hasofer and Lind, 1974) defined a reliability index as the shortest distance from the origin to the constraint in the standard normal space yielding a point-searching sub-problem:

$$
\begin{array}{ll}
\text { Min } & \left\|\boldsymbol{u}_{i}\right\| \\
\text { s.t. } & g_{i}\left(\boldsymbol{u}_{i}\right)=0
\end{array}
$$

The optimal solution of the Eq. (27) is called the Most Probable Failure Point (MPFP), $\boldsymbol{u}_{i}^{*}$, for the $i^{\text {th }}$ constraint. A modified reliability index (Lin, Gea et al., 2009), $\beta_{M}$ :

$\beta_{M, i}=\boldsymbol{u}_{i}^{*} \cdot \frac{\nabla_{u} g_{i}\left(\boldsymbol{u}_{i}^{*}\right)}{\left\|\nabla_{u} g\left(\boldsymbol{u}_{i}^{*}\right)\right\|}$

provides the appropriate evaluations of the failure probabilities anywhere in the design domain and it is constrained by the following inequality equation:

$-\beta_{M, i}(\boldsymbol{d}) \leq-\beta_{f, i}$

where $\beta_{f}$ is the allowable reliability index. Using the first-order Taylor expansion and the sensitivity analysis in (Lin, Gea et al., 2009), the probabilistic optimization problem is transformed to the following

$$
\begin{array}{ll}
\operatorname{Min}_{\boldsymbol{d}} & z(\boldsymbol{d}) \\
\text { s.t. } & -\boldsymbol{u}_{i}^{(k+1)} \cdot \nabla_{\boldsymbol{u}} g_{i}\left(\boldsymbol{u}_{i}^{(k+1)}\right)\left\|\nabla_{\boldsymbol{u}} g_{i}\left(\boldsymbol{u}_{i}^{(k+1)}\right)\right\|^{-1} \\
& +\left(\boldsymbol{d}-\boldsymbol{d}^{(k)}\right) \cdot \nabla_{\boldsymbol{d}} g_{i}\left(\boldsymbol{u}_{i}^{(k+1)}\right)\left\|\nabla_{\boldsymbol{u}} g_{i}\left(\boldsymbol{u}_{i}^{(k+1)}\right)\right\|^{-1} \\
& \leq-\beta_{f, i} \quad i=1 \ldots n \\
& \boldsymbol{d}^{L} \leq \boldsymbol{d} \leq \boldsymbol{d}^{U}
\end{array}
$$

Initially, the design variables, $\boldsymbol{d}^{(1)}$ and $\boldsymbol{u}^{(1)}$, are given. For the $k^{\text {th }}$ iteration, the MPFP is found by solving the Eq. (27) and updated to $\boldsymbol{u}^{(k+1)}$. In sequel, the Eq. (30) is solved to find the solution, $\boldsymbol{d}^{(k+1)}$. The iteration terminates when the absolute difference, $\left|\boldsymbol{d}^{(k)}-\boldsymbol{d}^{(k+1)}\right|$, is smaller than a small value, i.e. $10^{-3}$ in this study, or $k$ is larger than an allowable iteration number, i.e. 5 iterations (Yang and $\mathrm{Gu}, 2004$ ).

Performance Measure Approach (PMA): Alternatively, the failure probability can be evaluated by an inverse reliability analysis (Tu et al., 1999), while a target performance measure is given by

$\zeta_{i}(\boldsymbol{d})=F_{g_{i}}^{-1}\left\{1-\Phi\left[-\beta_{f, i}\right]\right\}$

where $F_{g_{i}}(\zeta)$ is the probability of the event of $g_{i}(\boldsymbol{X}) \leq \zeta$. Accordingly, the inequality constraint in Eq. (29) is transformed to the following equation

$\zeta_{i}(\boldsymbol{d}) \leq 0$

The target performance measure can be found at the maximum value of the performance constraint with the allowable reliability. Thus, a Most Probable Target Point (MPTP), $\boldsymbol{u}_{i}^{\#}$, is found by solving a subproblem

$$
\begin{array}{ll}
\text { Max } & g_{i}\left(\boldsymbol{u}_{i}\right) \\
\text { s.t. } & \left\|\boldsymbol{u}_{i}\right\|=\beta_{f, i}
\end{array}
$$

With the linear approximation of the Eq. (32), the solvable probabilistic formulation of the RBDO problem is then given as

$$
\begin{aligned}
\underset{\boldsymbol{d}}{\operatorname{Min}} & z(\boldsymbol{d}) \\
\text { s.t. } & g_{i}\left(\boldsymbol{u}_{i}^{(k+1)}\right)+\left(\boldsymbol{d}-\boldsymbol{d}^{(k)}\right) \cdot \nabla_{\boldsymbol{d}} g_{i}\left(\boldsymbol{u}_{i}^{(k+1)}\right) \leq 0 \quad i=1 \ldots n \\
& \boldsymbol{d}^{L} \leq \boldsymbol{d} \leq \boldsymbol{d}^{U}
\end{aligned}
$$

A similar optimization scheme is found in the PMA, while the MPTP must be firstly found by solving Eq. (33) for $k^{\text {th }}$ iteration and updated to $\boldsymbol{u}^{(k+1)}$. The Eq. (34) is then solved to find the better solution, $\boldsymbol{d}^{(k+1)}$, and the optimal solution is determined while the iteration terminates with the same convergence criterion. In this research, we mainly focus on the RIA from Eqs. (27) to (30). The optimization of the CVD processes using the PMA is discussed in (George et al., 2009).

RBDO with Non-Normally Distributed Random Variables: Not only can the MRIA be utilized to solve the typical RBDO problems with normally distributed random variables, but the methods to solve the ones with non-normally distributed random variables are investigated in this session. Famous non-normal distributions include lognormal, Weibull, Gumbel, and uniform distributions (Youn and Choi, 2004). For simplicity, the random variable, $\boldsymbol{X}$, is now considered to be independent and lognormally distributed and its $j^{\text {th }}$ component follows $X_{j} \sim \log N\left(d_{j}, \sigma_{j}\right)$. Using the transformation of $\boldsymbol{X}=\exp \boldsymbol{Y}$, an independent and normally distributed random variable, $\boldsymbol{Y}$, is obtained where its $j^{\text {th }}$ component follows $Y_{j} \sim N\left(d_{Y, j}, \sigma_{Y, j}\right)$ and these two equations: 


$$
\begin{aligned}
& \sigma_{Y, j}=\sqrt{\ln \left(1+\frac{\sigma_{j}^{2}}{d_{j}^{2}}\right)} \\
& d_{Y, j}=\ln d_{j}-\frac{1}{2} \sigma_{Y, j}^{2}
\end{aligned}
$$

Using $\boldsymbol{Y}=\boldsymbol{d}_{Y}+\boldsymbol{\sigma}_{Y} \cdot \boldsymbol{U}$, the transformation from the lognormal space to the standard normal space is established as $\boldsymbol{X}=\exp \left(\boldsymbol{d}_{Y}+\boldsymbol{\sigma}_{Y} \cdot \boldsymbol{U}\right)$.

The sub-problem in Eq. (27) is then solved to obtain the MPFP and the modified reliability index is given by the Eq. (28). The original probabilistic optimization problem with lognormally distributed random variables now becomes a solvable deterministic optimization problem as follows:

$$
\begin{array}{ll}
\operatorname{Min}_{\boldsymbol{d}_{Y}} & z\left(\boldsymbol{d}_{Y}\right) \\
\text { s.t. } & -\beta_{M, i}\left(\boldsymbol{d}_{Y}\right) \leq-\beta_{f, i}
\end{array}
$$

Notice that the nonlinear conversion from the lognormal design space to the standard normal space includes design-dependent parameters in Eqs. (35) and (36). These two parameters should be updated prior to the MPFP-searching sub-problem using the following two iterative schemes:

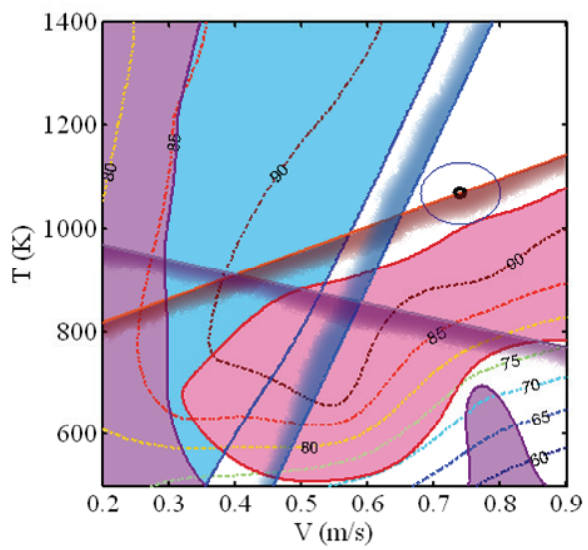

Fig. 14 Optimal solution of example $1 b$.
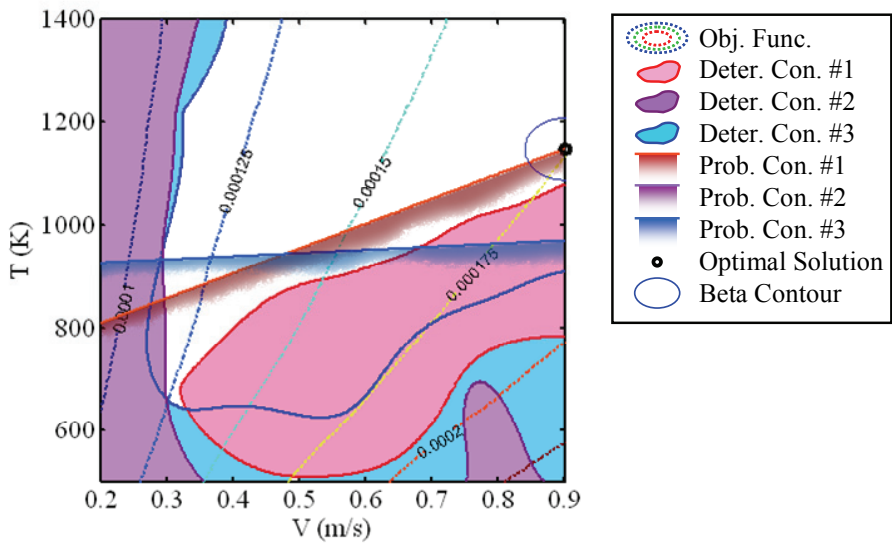

Fig. 15 Optimal solution of example 2b.

$$
\begin{aligned}
& \sigma_{Y, j}^{(k)}=\sqrt{\ln \left[1+\left(\sigma_{j}^{(k-1)} / d_{j}^{(k-1)}\right)^{2}\right]} \\
& d_{Y, j}^{(k)}=\ln d_{j}^{(k-1)}-0.5\left(\sigma_{Y, j}^{(k)}\right)^{2}
\end{aligned}
$$

These updating schemes do not cost any additional function calls of the performance constraints; however, the varying standard deviations do decrease the convergence efficiency of the RIA.

\subsection{RBDO of the CVD Process}

The described RBDO strategy, RIA with both normally and lognormally distributed random variables, are applied to the optimization formulations of the CVD processes in this session. The resultant failure probabilities will be verified by the Monte Carlo Simulations.

Example 1b - Maximizing the PWA Subject to Constraint of Deposition Rate with Normally Distributed Random Variables: Alterative to the Example 1a in Eq. (23), the failure probabilities of all the constraints are subjected to the $3 \sigma$ criteria The problem is given as

$$
\begin{array}{ll}
\underset{V, T}{\operatorname{Max}} & \text { PWA } \\
\text { s.t. } & P\left[\mathrm{RMS}>\mathrm{RMS}_{\mathrm{U}}\right] \leq 0.13 \% \\
& P\left[\mathrm{KUR}>\mathrm{KUR}_{\mathrm{U}}\right] \leq 0.13 \% \\
& P\left[\mathrm{MDR}_{\mathrm{L}}>\mathrm{MDR}\right] \leq 0.13 \% \\
& V_{L} \leq V \leq V_{U} \\
& T_{L} \leq T \leq T_{U}
\end{array}
$$

The standard deviations of the velocity and temperature are $0.02 \mathrm{~m} / \mathrm{s}$ and $20 \mathrm{~K}$ respectively and other problem settings remain the same.

Example 2b - Maximizing the MDR Subject to Constraint of Working Area with Normally Distributed Random Variables: Similarly, the Example 2a is reconsidered as the following

$$
\begin{array}{ll}
\underset{V, T}{\operatorname{Max}} & \text { MDR } \\
\text { s.t. } & P\left[\mathrm{RMS}>\mathrm{RMS}_{\mathrm{U}}\right] \leq 0.13 \% \\
& P\left[\mathrm{KUR}>\mathrm{KUR}_{\mathrm{U}}\right] \leq 0.13 \% \\
& P\left[\mathrm{PWA}_{\mathrm{L}}>\mathrm{PWA}\right] \leq 0.13 \% \\
& V_{L} \leq V \leq V_{U} \\
& T_{L} \leq T \leq T_{U}
\end{array}
$$

The Figs. 14 and 15 show the probabilistic constraints in Eq. (30). The resultant solutions are more conservative than the traditional deterministic optimization problems and the MCS results verify the acceptance of the failure probabilities of all the constraints, shown in Table 8.

Example 1c - Maximizing the PWA Subject to Constraint of Deposition Rate with Lognormally Distributed Random Variables: The optimization now maximizes the working area subject to the constraints of the uniformity and the deposition rate with the lognormally distributed random variables. The RBDO problem is formulated in Eq. (40) and the same considerations about the failure probabilities continue. The Fig. 16 demonstrates the probabilistic constraints and optimal solution in both the original space and the transformed normal space. The MCS results are shown in Table 8 . 


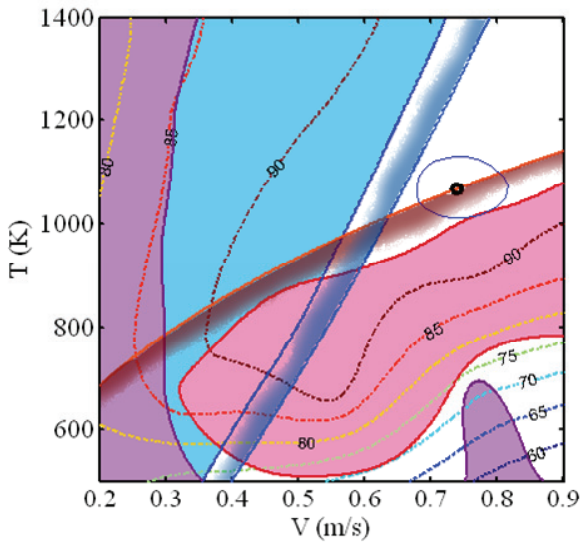

(a)
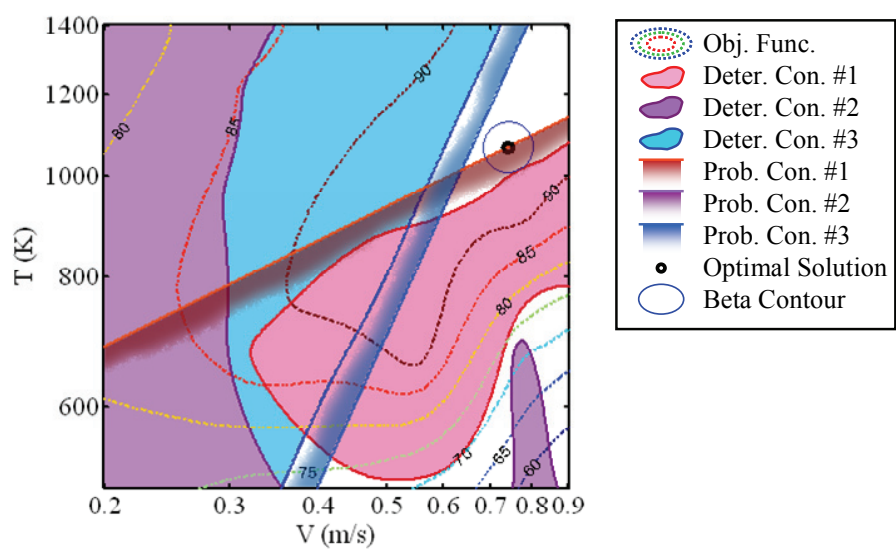

(b)

Fig. 16 Optimal solution of example 1c in (a) original lognormal and (b) transformed normal design space.

Example 2c - Maximizing the MDR Subject to Constraint of Working Area with Lognormally Distributed Random

Variables: The final example is to maximize the MDR subject to the constraints of the RMS, the KUR, and the PWA with the lognormally distributed inlet velocity and susceptor temperature. The problem formulation is shown in Eq. (41) and it is solved by the proposed updating scheme in Eqs. (38) and (39). The Fig. 17 shows the optimal solution in both lognormal and normal spaces and the MCS results are listed in Table 8.

Comparing the optimal solutions in both Tables 7 and 8, the MCS results have shown that the optimal solutions for the deterministic optimization formulations suffer from high probabilities of the system failures even though they are theoretically the best operating conditions for the CVD process without the design uncertainties. After considering the RBDO formulations and solving them by the RIA, more conservative operating conditions are found and the failure probabilities of all the constraints are satisfied within certain allowable range. The optimization problems of the CVD process with two different kinds of design uncertainties have been studied, including the normally and the lognormally distributed random variables. For the normally distributed random variables, the RBDO problem is solved directly by the RIA. On the other hand, the conversion to the normal space and the updating scheme of the varying standard deviations are necessary for non-normally distributed random variables.
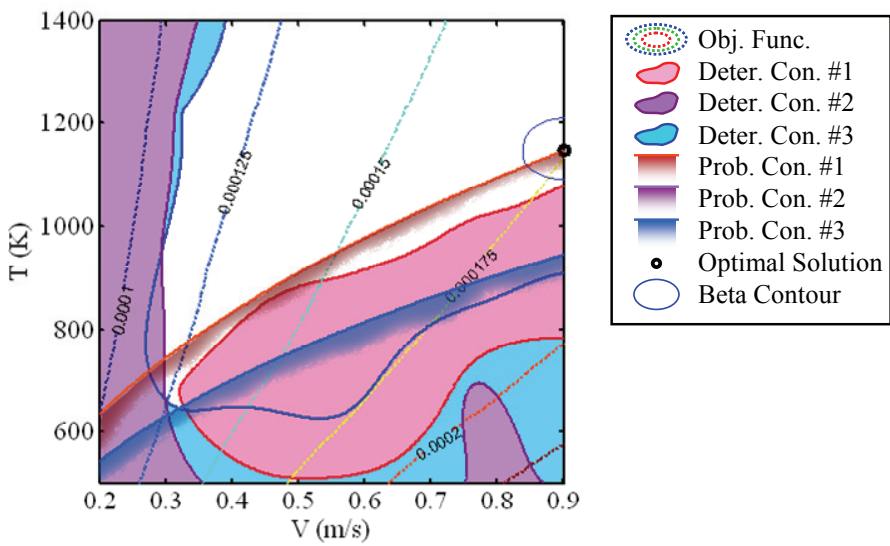

(a)
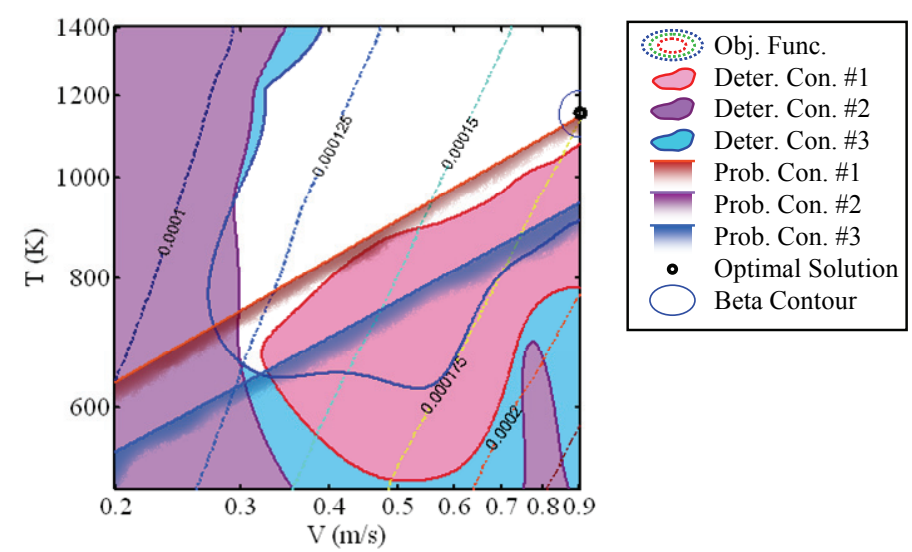

(b)

Fig. 17 Optimal solution of example 2c in (a) original lognormal and (b) transformed normal design space.

Table 8 The Monte Carlo Simulations for examples 1 and 2 with normally and lognormally distributed random variables.

\begin{tabular}{|c|c|c|c|c|c|}
\hline Ex. & $\begin{array}{c}\text { Distribution } \\
\text { of Uncertainty }\end{array}$ & $\begin{array}{c}\text { Optimal } \\
\text { Solution }\end{array}$ & $M C S_{1}$ & $M C S_{2}$ & $M C S_{3}$ \\
\hline 1b & Normal & $(0.74,1069)$ & $0.112 \%$ & $0 \%$ & $0 \%$ \\
\hline 1c & Lognormal & $(0.74,1067)$ & $0.118 \%$ & $0 \%$ & $0 \%$ \\
\hline 2b & Normal & $(0.90,1148)$ & $0.120 \%$ & $0 \%$ & $0 \%$ \\
\hline 2c & Lognormal & $(0.90,1147)$ & $0.110 \%$ & $0 \%$ & $0 \%$ \\
\hline
\end{tabular}

The RIA has been successfully applied to solve the RBDO problems of the CVD process with both normally and lognormally distributed random variables, the inlet velocity and the susceptor temperature. For normally distributed variables, the MPFPs are firstly found in the standard normal space and then utilized to approximate the probabilistic constraints. Those approximate probabilistic constraints are iteratively updated by the latest MPFPs with respect to the mean of the random variables. The global optimal operating conditions are found with the updated probabilistic constraints and the multiple starting points due to the concave feasible domains. For the nonnormally distributed random variables, the original design space is converted to the normal space and the variant standard deviations are iteratively updated before the RBDO problems are solved by the MRIA. The MCS results are utilized to verify the acceptance of the failure probabilities. 
The proposed systematic strategy of the optimization with consideration of design uncertainties can be applied to other thermal systems. For normally distributed random variables, the designer needs to measure the standard deviations and use the mean as the design variable. Transforming to the standard normal space, the MPFPs are determined for the constructions of the approximate probabilistic constraints. The optimal solution with acceptable reliability can then be found. Additionally, the standard deviations are updated iteratively for the non-normally distributed random variables.

\section{CONCLUSIONS}

A systematic strategy to parametric model and optimize of the thermal systems with design uncertainties has been proposed in this research work. The CVD has been chosen as an example, where the inlet velocity and the susceptor temperature are the design variables. The CVD process is simulated and the responses, the PWA, the MDR, the RMS, and the KUR, are utilized to represent the productivity and the uniformity of the thin-film deposition of silicon from silane. The RBF is utilized to model those responses. Then, the response surface models are used to formulate the optimization problems for finding the better operating conditions. The RIA is introduced to solve those optimization problems with both the normally and non-normally distributed random variables. In the optimization process of the RIA, the MPFPs are found and utilized to formulate the approximate probabilistic constraints. The optimal solution is finally found with the acceptable reliability. As a result, not only the proposed strategy can be utilized to model and optimize the CVD process with design uncertainties, but also it can be applied to other thermal systems.

The proposed strategy to simulate the thin-film growth of silicon, parametrically model the responses of the deposition, and optimize the operating condition with uncertainties is also very useful for other thinfilm fabrications in semiconductors. One of the examples is the spincoating process of polymers on the silicon wafer, which is a critical post-process of photolithography (Jaeger, 2002). The design variables include the velocity of the spin-coater, the position of the silicon wafer, and the amount of the applied polymers. The responses include the thickness and the uniformity of the polymer thin-film, which can be modeled by the RBF in terms of experiment data. The proposed method can be utilized to find the optimal operating conditions for the desired thickness with the allowable uniformity in terms of the root mean square and the surface kurtosis. In the optimization process of the thin-film growth, the design uncertainties in the design variables, including the spin velocity and the position of the water, should be considered. The RIA provides the evaluations of the failure probabilities and generates the approximate probabilistic constraints. The optimization problems with design uncertainties can then solved and the optimal operating condition can be obtained. Similarly, the proposed strategy can be directly applied to any other thin-film growth of the thermal systems.

Besides the thermal systems with thin-film growth, the proposed method can also be applied to other thermal systems, such as the cooling design of the heat sink on the CPU chip of a computer. In the example of the cooling design of the heat sink, the fluid mechanics and heat transfer is different from the CVD process. Either experiments or numerical simulations should be designed with the different hardware parameters or operating conditions. The hardware parameters include the dimension of the heat sink, the dimension of the fin, the orientation of the heat sink, and the working space of the desktop case; on the other hand, the operating conditions include the temperature of the CPU chip and the velocity of the cooling fan in the case. Collecting the data from the experiments/simulations, some responses need to be defined to quantitatively describe the performance of the design. The responses are then modeled parametrically by the Radial Basis Functions and utilized to formulate the optimization problems. The recognitions of the design uncertainties and the distributions of the random variables are also important for the RBDO formulations. Once the probabilistic optimization problems are formulated, the RIA can be used to find optimal designs with allowable failure probabilities. To sum up, the following summarize the key steps for applying the proposed method to design and optimize other thermal systems with design uncertainties:

- The design and execution of the experiments or the simulations.

- The definition of the responses.

- The formulation of the optimization problems.

- The recognition of the design uncertainties.

With the achievements of the above four key steps, the proposed system strategy can be applied to the specific thermal systems and find the optimal design variables with design uncertainties.

The proposed strategy of parametric modeling and optimization with design uncertainties can be applied to many other thermal systems. In the other study of the CVD process, the proposed methodology can be applied to find the optimal operating conditions for the depositions of different materials, such as the high-hardness materials - boron nitride $(\mathrm{BN})$, silicon carbide $(\mathrm{SiC})$, boron carbide $\left(\mathrm{B}_{4} \mathrm{C}\right)$, and titanium nitride (TiN). Different reaction kinetics of the deposition should be studied. Different configurations of the CVD processes also can be considered with different hardware variables, such as the shape of the CVD chamber, the orientation of the susceptor, and the direction of the reactant flow. Alternatively, the thermal systems that are related to the safety of human lives, the energy crisis, and the biotechnology are very important. Especially for the system that requires high performance and cannot afford system failures, the operating conditions of the thermal systems should be determined by the proposed method. One of the examples is the next-generation design of the carbon fuel cells (Steinberg et al., 2002), where the efficiency for electricity production may be possibly higher than the traditional hydrogen fuel cells. The operating conditions of producing the carbon fuel cells differ the disorderedness of the carbon atoms, which is much related to the electricity yield.

\section{ACKNOWLEDGEMENTS}

The authors would like to acknowledge the useful personal discussions with Dr. Liping Wang at General Electric about the utilization of the Radial Basis Function, as well as the treasurable suggestions from Dr. Kyung K. Choi at University of Iowa about the designs with nonnormally distributed random variables.

\section{NOMENCLATURE}

$A \quad$ The pre-exponential factor for Arrhenius rate.

A A $M \times K$ matrix of monomials for the RSM;

$$
\boldsymbol{A}\left(\boldsymbol{x}^{S}\right)=\sum_{s=1}^{M} \sum_{t=1}^{K} B_{t}\left(\boldsymbol{x}_{s}^{S}\right) \boldsymbol{e}_{s} \boldsymbol{e}_{t} .
$$

B A combination of the monomials for the RSM;

$$
\boldsymbol{B}(\boldsymbol{x})=B_{t}(\boldsymbol{x}) \boldsymbol{e}_{t} \text {. }
$$

$C_{P} \quad$ Specific heat in the governing equations.

$C_{x} \quad$ A vector of the covariance functions for Kriging;

$$
\boldsymbol{C}_{x}\left(\boldsymbol{x}, \boldsymbol{x}^{S}\right)=\sum_{s=1}^{M} \operatorname{Cov}\left(\boldsymbol{x}, \boldsymbol{x}_{s}^{S}\right) \boldsymbol{e}_{s} .
$$

C A matrix of the covariance functions for Kriging;

$$
\boldsymbol{C}\left(\boldsymbol{x}^{S}\right)=\sum_{r=1}^{M} \sum_{s=1}^{M} \operatorname{Cov}\left(\boldsymbol{x}_{r}^{S}, \boldsymbol{x}_{s}^{S}\right) \boldsymbol{e}_{r} \boldsymbol{e}_{s} .
$$

$\boldsymbol{d}$ The expected value of $\boldsymbol{X} ; \boldsymbol{d}=E[\boldsymbol{X}]=d_{j} \boldsymbol{e}_{j}$.

$\boldsymbol{d}_{Y} \quad$ The expected value of $\boldsymbol{Y} ; \boldsymbol{d}_{Y}=E[\boldsymbol{Y}]=d_{Y, j} \boldsymbol{e}_{j}$.

$D \quad$ Mass diffusivity in the governing equations. 
$D_{0} \quad$ The pre-exponential factor in the power law of the mass diffusivity.

$D_{l} \quad$ Deposition rate at the $t^{\text {th }}$ sampling node.

$\boldsymbol{e} \quad$ Normal basis of the design variables.

$E_{a} \quad$ The activation energy for Arrhenius rate.

$f(\boldsymbol{x})$ The JPDF of $g(\boldsymbol{X})$.

$\vec{F} \quad$ Body force in the governing equations.

$F(\boldsymbol{x})$ An approximate function by RSM.

$F_{\eta} \quad$ The function value of a RSM model at $\boldsymbol{x}_{\eta} ; F_{\eta}=F\left(\boldsymbol{x}_{\eta}\right)$.

$\boldsymbol{F}^{S} \quad$ A vector of the responses in terms of the sampling points; $\boldsymbol{F}^{S}\left(\boldsymbol{x}^{S}\right)=\sum_{s=1}^{M} F\left(\boldsymbol{x}_{s}^{S}\right) \boldsymbol{e}_{s}$.

$F_{g}(\gamma)$ The CDF of the event of $g \leq \gamma$.

$G_{\eta} \quad$ The simulated response in terms of $\boldsymbol{x}_{\eta}$.

$g$ Performance constraint; $g(\boldsymbol{X})>0$ is considered as the failed design and $g(\boldsymbol{X}) \leq 0$ represents the feasible domain.

$k \quad$ The iteration number of the global optimization loop.

$k_{T} \quad$ Thermal diffusivity in the governing equations.

$K \quad$ The number of the parameters to be determined in a best fitting model; $K<M$.

$m \quad$ Mass fraction of species in the governing equations.

$M \quad$ The number of the sampling points.

$n \quad$ The number of the constraints.

$N \quad$ The number of the design variables.

$p \quad$ Pressure in the governing equations.

$P \quad$ The probability of an event.

$Q \quad$ The number of the uniformly distributed sampling nodes within the effective working area.

$\dot{Q} \quad$ Heat source in the governing equations.

$R \quad$ Production rate of species due to chemical reactions.

$R_{g} \quad$ The universal gas constant.

$S \quad$ The local slope between two nodes in the deposition profile from the numerical simulation.

$T \quad$ Temperature in the governing equations; the susceptor temperature in the optimization of CVD processes. The deterministic standard normal design variable; $\boldsymbol{u}=u_{j} \boldsymbol{e}_{j}$ and $u_{j}=\left(x_{j}-d_{j}\right) / \sigma_{j}$.

$\boldsymbol{u}^{*} \quad$ The MPFP; $\boldsymbol{u}^{*}=u_{j}^{*} \boldsymbol{e}_{j}$.

$\boldsymbol{u}^{\#} \quad$ The MPTP; $\boldsymbol{u}^{\#}=u_{j}^{\#} \boldsymbol{e}_{j}$.

$\boldsymbol{U}$ The random standard normal design variable; $\boldsymbol{U}=U_{j} \boldsymbol{e}_{j}$.

$\vec{v} \quad$ Fluid velocity in the governing equations.

$V \quad$ The inlet velocity of the CVD process.

$\boldsymbol{w}$ The coefficients of the RSM models; $\boldsymbol{w}=w_{r} \boldsymbol{e}_{r}$ for the exact fitting and $\boldsymbol{w}=w_{t} \boldsymbol{e}_{t}$ for the best fitting.

$\boldsymbol{x} \quad$ The deterministic design variable; $\boldsymbol{x}=x_{j} \boldsymbol{e}_{j}$.

$\boldsymbol{X}$ The random design variable; $\boldsymbol{X}=X_{j} \boldsymbol{e}_{j}$.

$\boldsymbol{y}$ The deterministic design variable; $\boldsymbol{y}=y_{j} \boldsymbol{e}_{j}$.

$\boldsymbol{Y}$ The random design variable; $\boldsymbol{Y}=Y_{j} \boldsymbol{e}_{j}$.

$z \quad$ The cost function.

err The error measurement for the model validation.

EWA The effective working area.

KUR The Surface kurtosis of the deposition profile in the EWA.
MDR The mean of the deposition rate in the EWA.

PWA The percentage of the working area.

RMS The root mean square of the deposition profile in the EWA.

$\begin{array}{ll}\operatorname{Cov}\left(X_{p}, X_{q}\right) & \text { Covariance between } X_{p} \text { and } X_{q} . \\ \operatorname{Cov}\left(\boldsymbol{x}_{r}, \boldsymbol{x}_{s}\right) & \text { Covariance between two vectors: } \boldsymbol{x}_{r}, \boldsymbol{x}_{s} \in R^{N} . \\ \log N(\mu, \sigma) & \text { A lognormal distribution with the mean, } \mu, \text { and }\end{array}$
the standard deviation, $\sigma$.

$N(\mu, \sigma) \quad$ A normal distribution with the mean, $\mu$, and the standard deviation, $\sigma$.

Greek Symbols

$\alpha \quad$ The temperature exponent for the Arrhenius rate.

$\beta \quad$ Reliability index.

$\gamma \quad$ The temperature exponent for the mass diffusivity of the silane.

$\theta \quad$ Unknown parameters for the Gaussian correlation functions.

$\kappa \quad$ The Arrhenius rate.

$\lambda$ The parameter for the smoothness of the distance function.

$\mu \quad$ Dynamic viscosity in the governing equations.

$\zeta \quad$ Performance measure.

$\rho \quad$ Density in the governing equations.

$\boldsymbol{\sigma} \quad$ The standard deviation of $\boldsymbol{X} ; \boldsymbol{\sigma}=\sum_{j=1}^{N} \sigma_{j} \boldsymbol{e}_{j} \boldsymbol{e}_{j}$.

$\boldsymbol{\sigma}_{Y} \quad$ The standard deviation of $\boldsymbol{Y} ; \boldsymbol{\sigma}_{Y}=\sum_{j=1}^{N} \sigma_{Y, j} \boldsymbol{e}_{j} \boldsymbol{e}_{j}$

$\Phi \quad$ The Standard Normal CDF.

Superscripts

(k) $\quad k^{\text {th }}$ Iteration of the global optimization loop.

$S \quad$ Quantities evaluated at the sampling points.

* Optimal solution.

Subscripts

$f \quad$ Allowable level of a quantity.

$h \quad$ The index of the important sampling points; $0<$ size of $h<N$.

$i \quad$ The dimensional index of the constraint; $i=1,2, \ldots, n$.

$j$ The dimensional index of the design variable; $j=1,2, \ldots, N$.

$L \quad$ Lower bound.

$M \quad$ Modified reliability index.

$m \quad$ Maximum value.

$p \quad$ The index of the design variable for the model validation; $\eta=1,2, \ldots, 437$.

$q \quad$ The index of the uniformly distributed sampling nodes; $q=1,2, \ldots, Q$.

$r \quad$ A dummy index; $r=1,2, \ldots, M$.

$s \quad$ A dummy index; $s=1,2, \ldots, M$.

$t \quad$ A dummy index; $t=1,2, \ldots, K$.

$U \quad$ Upper bound.

\section{REFERENCES}

Allen, S.D., 1981, "Laser Chemical Vapor Deposition: A Technique for Selective Area Deposition," Journal of Applied Physics, 52(11), 65016505.

doi: $10.1063 / 1.328600$

Breiland, W.G. and Coltrin, M.E., 1990, "Si Deposition Rates in a TwoDimensional CVD Reactor and Comparisons with Model Calculations," Journal of The Electrochemical Society, 137, 2313-2319. doi: 10.1149/1.2086933 
Cheng, H.-E., Chiang, M.-J., and Han, M.-H., 1995, "Growth Characteristics and Properties of TiN Coating by Chemical Vapor Deposition," Journal of The Electrochemical Society, 142, 1573-1578. doi: 10.1149/1.2048615

Chiu, W.K.S., 1999, Simulation, Design and Optimization of Chemical Vapor Deposition systems for Advanced Materials, Dissertation, Mechanical and Aerospace Engineering, Rutgers University.

Chiu, W.K.S. and Jaluria, Y., 1997, "Heat Transfer in Horizontal and Vertical CVD Reactors," ASME Heat Transfer Divison, 347, 293-311.

Chiu, W.K.S. and Jaluria, Y., 1999, "Effect of Buoyancy, Susceptor Motion, and Conjugate Transport in Chemical Vapor Deposition Systems," Journal of Heat Transfer, 121, 757-761.

doi: $10.1115 / 1.2826049$

Chiu, W.K.S. and Jaluria, Y., 2000, "Continuous Chemical Vapor Deposition Processing with a Moving Finite Thickness Susceptor," Journal of Materials Research, 15, 317-328.

doi: 10.1557/JMR.2000.0050

Chiu, W.K.S., Jaluria, Y., and Glumac, N.G., 2000, "Numerical Simulation of Chemical Vapor Deposition Processes Under Variable and Constant Property Approximations," Numerical Heat Transfer, 37, 113-132.

doi: $10.1080 / 104077800274334$

Chiu, W.K.S., Jaluria, Y., and Glumac, N.G., 2002, "Control of Thin Film Growth In Chemical Vapor Deposition Manufacturing Systems: A Feasibility Study," Journal of Manufacturing Science and Engineering, 124, 715-724.

doi: $10.1115 / 1.1465434$

Chiu, W.K.S., Richards, C.J., and Jaluria, Y., 2000, "Flow Structure and Heat Transfer in a Horizontal Converging Channel Heated From Below," Physics of Fluids, 12, 2128-2136.

doi: $10.1063 / 1.870458$

Chiu, W.K.S., Richards, C.J., and Jaluria, Y., 2001, "Experimental and Numerical Study of Conjugate Heat Transfer in a Horizontal Channel Heated From Below," Journal of Heat Transfer, 123, 688-697.

doi: $10.1115 / 1.1372316$

Creighton, J.R. and Parmeter, J.E., 1993, "Metal CVD for Microelectronic Applications: An Examination of Surface Chemistry and Kinetics," Critical Reviews in Solid State and Materials Sciences, 18, $175-238$. doi: $10.1080 / 10408439308242560$

Den Hertog, D., Kleijnen, J.P.C., and Siem, A.Y.D., 2006, "The Correct Kriging Variance Estimated by Bootstrapping," Journal of the Operational Research Society, 57(4), 400-409.

doi: $10.1057 /$ palgrave.jors. 2601997

Dimitrios, I., Kremer, A.M., McKenna, D.R., and Jensen, K.F., 1987, "Complex Flow Phenomena in Vertical MOCVD Reactors: Effects on Deposition Uniformity and Interface Abruptness," Journal of Crystal Growth, 85, 154-164.

Duchon, J., 1977, "Splines Minimizing Rotation-Invariant Semi-Norms in Sobolev Spaces," Schempp, W. and Zeller, K. eds., Constructive Theory of Functions of Several Variables, Springer Berlin / Heidelberg, 85-100.

doi: $10.1007 / \mathrm{BFb} 0086566$

Eden, J.G., 1991, "Photochemical Vapor Deposition," Vossen, J.L. and Kern, W. eds., Thin Film Processes II, Academic Press, Inc., San Diego, CA.

Eversteyn, F.C., Severin, P.J.W., van den Brekel, C.H.J., and Peek, H.L., 1970, "A Stagnant Layer Model for the Epitaxial Growth of
Silicon from Silane on a Horizontal Reactor," Journal of The Electrochemical Society, 117, 925-931.

doi: $10.1149 / 1.2407685$

Fotiadis, D.I., Boekholt, M., Jensen, K.F., and Richter, W., 1990, "Flow and Heat Transfer in CVD Reactors: Comparison of Raman Temperature Measurements and Finite Element Predictions," Journal of Crystal Growth, 100, 577-599.

doi: 10.1016/0022-0248(90)90257-L

Fox, R.W. and McDonald, A.T., 1992, Introduction to Fluid Mechanics, $4^{\text {th }}$ ed., Wiley, New York.

Foxon, C.T., 1994, "Principles of Molecular Beam Epitaxy," Hurle, D. ed. Handbook of Crystal Growth, Elsevier, Amsterdam.

Fraser, D.B., 1983, "Metallization," Sze, S.M. ed. VLSI Technology, McGraw-Hill, New York, 347.

Gardeniers, J.G.E., Maas, W.E.J.R., Van Meerten, R.Z.C., and Giling, L.J., 1989, "Influence of Temperature on the Crystal Habit of Silicon in the Si-H-Cl CVD System: I. Experimental Results," Journal of Crystal Growth, 96, 821-842.

doi: 10.1016/0022-0248(89)90642-8

Gebhart, B., Jaluria, Y., Mahajan, R.L., and Sammakia, B., 1988, Buoyancy-Induced Flows and Transport, Taylor \& Francis, Philadelphia, PA.

George, P., 2007, Simulation and Optimization of the Chemical Vapor Deposition Process, Dissertation, Mechanical and Aerospace Engineering, Rutgers University.

George, P., Lin, P.T., Gea, H.C., and Jaluria, Y., 2009, "ReliabilityBased Optimisation of Chemical Vapour Deposition Process," International Journal of Reliability and Safety, 3(4), 363-383.

Giesl, P., 2007, "Construction of Global Lyapunov Functions Using Radial Basis Functions," J.-M. Morel, C., F. Takens, G., and B. Teissier, P. eds., Lecture Notes in Mathematics, Springer-Verlag Berlin Heidelberg.

Giling, L.J., 1982, "Gas Flow Patterns in Horizontal Epitaxial Reactor Cells Observed by Interference Holography," Journal of The Electrochemical Society, 129(3), 634-644.

doi: $10.1149 / 1.2123939$

Gladfelter, W.L., 1993, "Selective Metallization by Chemical Vapor Deposition," Chemistry of Materials, 5, 1372-1388.

Goodwin, D.G., 1993, "Scaling Laws for Diamond Chemical Vapor Deposition. I. Diamond Surface Chemistry," Journal of Applied Physics, 74(11), 6888-6894.

doi: $10.1063 / 1.355063$

Gray, D.D. and Giorgini, A., 1976, "The Validity of the Boussinesq Approximation for Liquids and Gases," International Journal of Heat and Mass Transfer, 19, 545-551.

doi: 10.1016/0017-9310(76)90168-X

Hasofer, A.M. and Lind, N.C., 1974, "Exact and Invariant SecondMoment Code Format," Journal of the Engineering Mechanics Division, 100(EM1), 111-121.

Hintermann, H.E., 1996, "Advances and Development in CVD Technology," Materials Science and Engineering, A209, 366-371. doi: 10.1016/0921-5093(95)10131-4

Jaeger, R.C., 2002, Introduction to Microelectronic Fabrication, $2^{\text {nd }}$ ed., Prentice-Hall, Inc., Upper Saddle River, New Jersey.

Jaluria, Y., 1998, Design and Optimization of Thermal Systems, McGraw-Hill. 
Jensen, K.F., Einset, E.O., and Fotiadis, D.I., 1991, "Flow Phenomena in Chemical Vapor Deposition of Thin Films," Annual Review of Fluid Mechanics, 23, 197-232.

doi: 10.1146/annurev.f1.23.010191.001213

Jeong, S., Murayama, M., and Yamamoto, K., 2004, "Efficient Optimization Design Method Ssing Kriging Model," $42^{\text {nd }}$ AIAA Aerospace Sciences Meeting and Exhibit, Reno, NV.

Karki, K.C., Sathyamurthy, P.S., and Patankar, S.V., 1993, "ThreeDimensional Mixed Convection in a Horizontal Chemical Vapor Deposition Reactor," Journal of Heat Transfer, 115(3), 803-806. doi: $10.1115 / 1.2910760$

Kays, W.M. and London, A.L., 1984, Compact Heat Exchangers, McGraw-Hill, New York.

Kee, R.J., Ting, A., and Spence, P.A., 1995, "Understanding and Improving Materials Processing Through Interpreting and Manipulating Predictive Models," Chemical Vapor Deposition of Refractory Metals and Ceramics III, Materials Research Society, 363, 314.

Kreider, J.F. and Rabl, A., 1994, Heating and Cooling of Buildings: Design for Efficiency, McGraw-Hill, New York.

Kurtz, S., Friedman, D., Geisz, J., and McMahon, W., 2007, "Using MOVPE Growth to Generate Tomorrow's Solar Electricity," Journal of Crystal Growth, 298, 748-753.

doi: 10.1016/j.jcrysgro.2006.10.176

Lau, K.K.S., Lewis, H.G.P., Limb, S.J., Kwan, M.C., and Gleason, K.K., 2001, "Hot-Wire Chemical Vapor Deposition (HWCVD) of Fluorocarbon and Organosilicon Thin Films," Thin Solid Films, 395(12), 288-291.

doi: 10.1016/S0040-6090(01)01287-1

Lebensztajn, L., Marretto, C.A.R., Costa, M.C., and Coulomb, J.-L., 2004, "Kriging: A Useful Tool for Electromagnetic Device Optimization," IEEE Transaction on Magnetics, 40(2), 1196-1199. doi: 10.1109/TMAG.2004.824542

Lin, P.T., 2010, Parametric Modeling and Optimization of Thermal Systems with Design Uncertainties, Dissertation, Mechanical and Aerospace Engineering, Rutgers University.

Lin, P.T., Gea, H.C., and Jaluria, Y., 2009, "A Modified Reliability Index Approach for Reliability-Based Design Optimization," DETC2009-87804, 2009 ASME International Design Engineering Technical Conferences \& Computers and Information in Engineering Conference (IDETC/CIE), San Diego, CA, USA.

Lin, P.T., Jaluria, Y., and Gea, H.C., 2008, "Parametric Modeling and Optimization of Chemical Vapor Deposition Process," DETC200850054, 2008 ASME International Design Engineering Technical Conferences \& Computers and Information in Engineering Conference (IDETC/CIE), New York City, NY, USA.

Lin, P.T., Jaluria, Y., and Gea, H.C., 2009, "Parametric Modeling and Optimization of Chemical Vapor Deposition Process," Journal of Manufacturing Science and Engineering, 131(1), 011011.

doi: $10.1115 / 1.3063689$

Ly, H.V. and Tran, H.T., 2002, "Proper Orthogonal Decomposition for Flow Calculations and Optimal Control in a Horizontal CVD Reactor," Quarterly of Applied Mathematics, 60(4), 631-656.

Mahajan, R.L., 1996, "Transport Phenomena in Chemical Vapor Deposition Systems," Advances in Heat Transfer, Academic Press, 339425.
Mahajan, R.L. and Wei, C., 1991, "Buoyancy, Soret, Dufour, and Variable Property Effects in Silicon Epitaxy," Journal of Heat Transfer, 113, 688-695.

Mitchell, T.O., Hoyt, J.L., and Gibbons, J.F., 1997, "Substitutional Carbon Incorporation in Epitaxial $\mathrm{Si}_{1-\mathrm{y}} \mathrm{C}_{\mathrm{y}}$ Layers Grown by Chemical Vapor Deposition," Applied Physics Letters, 71(12), 1688-1690. doi: $10.1063 / 1.119794$

Moffat, H. and Jensen, K.F., 1986, "Complex Flow Phenomena in MOCVD Reactors: I. Horizontal Reactors," Journal of Crystal Growth, 77, 108-119.

doi: 10.1016/0022-0248(86)90290-3

Mortenson, M.E., 2006, Geometric Modeling, $3^{\text {rd }}$ ed., Industrial Press Inc., New York.

Mouche, M.-J., Mermet, J.-L., Pires, F., Richard, E., Torres, J., Palleau, J., and Braud, F., 1995, "Process Optimization of Copper MOCVD Using Modeling Experimental Design," Applied Surface Science, 91, 129-133.

doi: $10.1016 / 0169-4332(95) 00107-7$

Myers, R.H., Montgomery, D.C., and Anderson-Cook, C.M., 2009, Response Surface Methodology: Process and Product Optimization Using Designed Experiments, $3^{\text {rd }}$ ed., John Wiley \& Sons, Inc., Hoboken, New Jersey.

Naslain, R. and Langlais, F., 1986, "CVD Processing of CeramicCeramic Composite Materials," Materials Science Research, 20, 145.

Nilsen, O., 2003, Growth of Thin Films of Functional Oxides with the ALCVD Method, Doctor Scientiarium, Department of Chemistry, Faculty of Mathematics and Natural Sciences, University of Oslo, Norway.

Patankar, S.V., 1980, Numerical Heat Transfer and Fluid Flow, Hemisphere Publishing Corporation.

Quazzani, J., Chiu, K.C., and Rosenberger, F., 1988, "On the 2D Modelling of Horizontal CVD Reactors and Its Limitations," Journal of Crystal Growth, 91, 497-508.

doi: 10.1016/0022-0248(88)90117-0

Quazzani, J. and Rosenberger, F., 1990, "Three- Dimensional Modeling of Horizontal Chemical Vapor Deposition I. MOCVD at Atmospheric Pressure," Journal of Crystal Growth, 100, 545-576. doi: 10.1016/0022-0248(90)90256-K

Raja, L.L., Kee, R.J., Serban, R., and Petzold, L.R., 2000, "Computational Algorithm for Dynamic Optimization of Chemical Vapor Deposition Processes in Stagnation Flow Reactors," Journal of The Electrochemical Society, 147(7), 2718-2726.

doi: 10.1149/1.1393595

Rebenne, H.E. and Bhat, D.G., 1994, "Review of CVD TiN Coatings for Wear-Resistant Applications: Deposition Processes, Properties and Performance," Surface and Coatings Technology, 63, 1-13. doi: 10.1016/S0257-8972(05)80002-7

Sherman, A., 1987, Chemical Vapor Deposition for Microelectronics, Noyes Publications, Park Ridge, New Jersey, USA.

Simpson, T.W., Peplinski, J.D., Koch, P.N., and Allen, J.K., 2001, "Metamodels for Computer-Based Engineering Design: Survey and Recommendations," Engineering with Computers, 17, 129-150. doi: 10.1007/PL00007198.

Southwell, R.P., Mendicino, M.A., and Seebauer, E.G., 1996, "Optimization of Selective $\mathrm{TiSi}_{2}$ Chemical Vapor Deposition by Mechanistic Chemical Kinetics," The Journal of Vacuum Science and Technology A, 14(3), 928-934. 


\section{doi: $10.1116 / 1.580417$}

Spall, R.E., 1996, "Unsteady Mixed Convection in Horizontal Ducts with Applications to Chemical Vapor Deposition Processes," International Communications in Heat and Mass Transfer, 23(1), 115122.

\section{doi: 10.1016/0735-1933(95)00089-5}

Stein, M., 1987, "Large Sample Properties of Simulations Using Latin Hypercube Sampling," Technometrics, 29(2), 143. doi: $10.2307 / 1269769$

Steinberg, D.S., 1980, Cooling Techniques for Electronic Equipment, Wiley-Interscience, New York.

Stoecker, W.F. and Jones, J.W., 1982, Refrigeration and Air Conditioning, $2^{\text {nd }}$ ed., McGraw-Hill, New York.

Stringfellow, G.B., 2001, "Fundamental Aspects of Organometallic Vapor Phase Epitaxy," Materials Science and Engineering, B87, 97116.

doi: 10.1016/S0921-5107(01)00712-7

Sugawara, K., 1972, "Silicon Epitaxial Growth by Rotating Disk Method," Journal of The Electrochemical Society, 119(12), 1749-1760. doi: 10.1149/1.2404093

Tu, J., Choi, K.K., and Park, Y.H., 1999, "A New Study on Reliability Based Design Optimization," Journal of Mechanical Design, 121, 557564.

doi: $10.1115 / 1.2829499$

Van Beers, W.C.M. and Kleijnen, J.P.C., 2003, "Kriging for Interpolation in Random Simulation," Journal of the Operational Research Society, 54(3), 255-262.

doi: $10.1057 /$ palgrave.jors.2601492

Van Beers, W.C.M. and Kleijnen, J.P.C., 2004, "Kriging Interpolation in Simulation: A Survey," Ingalls, R.G., Rossetti, M.D., Smith, J.S., and Peters, B.A. eds., Proceedings of the $36^{\text {th }}$ Conference on Winter Simulation, 113-121.

Van Wylen, G.J. and Sonntag, R.E., 1986, Fundamentals of Classical Thermodynamics, $3^{\text {rd }}$ ed., Wiley, New York.

Visser, E.P., Kleijn, C.R., Govers, C.A.M., Hoogendoorn, C.J., and Giling, L.J., 1989, "Return Flows in Horizontal MOCVD Reactors Studied with the Use of $\mathrm{TiO} 2$ Particle Injection and Numerical Calculations," Journal of Crystal Growth, 94, 924-946.

\section{doi: $10.1016 / 0022-0248(89) 90127-9$}

Wahl, G., 1977, "Hydrodynamic Description of CVD (Chemical Vapour Deposition) Processes," Thin Solid Films, 40(1-3), 13-26.

doi: 10.1016/0040-6090(77)90099-2

Wang, C.A., Groves, S.H., Palmateer, S.C., Weyburne, D.W., and Brown, R.A., 1986, "Flow Visualization Studies for Optimization of OMVPE Reactor Design " Journal of Crystal Growth, 77(1-3), 136143.

doi: 10.1016/0022-0248(86)90293-9

Wang, Q., Yoo, H., and Jaluria, Y., 2003, "Convection in a Horizontal Duct under Constant and Variable Property Formulations," International Journal of Heat and Mass Transfer, 46, 297-310. doi: 10.1016/S0017-9310(02)00272-7

Wendland, H., 1995, "Piecewise Polynomial, Positive Definite and Compactly Supported Radial Functions of Minimal Degree," Advances in Computational Mathematics, 4(1), 389-396. doi: 10.1007/BF02123482

Yang, R.J. and Gu, L., 2004, "Experience with Approximate Reliability-Based Optimization Methods," Structural and Multidisciplinary Optimization, 26(2), 152-159.

doi: 10.1007/s00158-003-0319-2

Yang, Y.-T. and Peng, H.-S., 2008, "Numerical Study of Pin-Fin Heat Sink with Un-Uniform Fin Height Design," International Journal of Heat and Mass Transfer, 51(19-20), 4788-4796. doi: 10.1016/j.ijheatmasstransfer.2008.02.017

Yang, Y.-T. and Peng, H.-S., 2009, "Numerical Study of the Heat Sink with Un-Uniform Fin Width Designs," International Journal of Heat and Mass Transfer, 52(15-16), 3473-3480.

doi: 10.1016/j.ijheatmasstransfer.2009.02.042

Yoo, H. and Jaluria, Y., 2002, "Thermal Aspects in the Continuous Chemical Vapor Deposition in Silicon," Journal of Heat Transfer, 124, 938-946.

doi: $10.1115 / 1.1482084$

Youn, B.D. and Choi, K.K., 2004, "An Investigation of Nonlinearity of Reliability-Based Design Optimization Approaches," Journal of Mechanical Design, 126(3), 403-411.

doi: $10.1115 / 1.1701880$ 